\title{
Report from the Photopolymer Additive Manufacturing Workshop: Roadmapping a Future for Stereolithography, Inkjet, and Beyond
}

Callie Higgins

Jason Killgore

Dianne Poster 


\title{
Report from the Photopolymer Additive Manufacturing Workshop: Roadmapping a Future for \\ Stereolithography, Inkjet, and Beyond
}

\author{
Callie Higgins \\ Jason Killgore \\ Nanoscale Reliability Group \\ Material Measurement Laboratory \\ Dianne Poster \\ Material Measurement Laboratory
}

This publication is available free of charge from:

https://doi.org/10.6028/NIST.SP.1500-17

January 2021

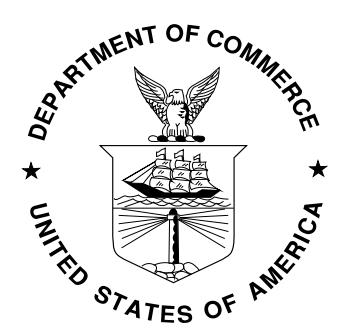

U.S. Department of Commerce Wilbur L. Ross, Jr., Secretary

National Institute of Standards and Technology Walter Copan, NIST Director and Undersecretary of Commerce for Standards and Technology 
Certain commercial entities, equipment, or materials may be identified in this document in order to describe an experimental procedure or concept adequately. Such identification is not intended to imply recommendation or endorsement by the National Institute of Standards and Technology, nor is it intended to imply that the entities, materials, or equipment are necessarily the best available for the purpose.

Publications in the SP1500 subseries are intended to capture external perspectives related to NIST standards, measurement, and testing-related efforts. These external perspectives can come from industry, academia, government, and others. These reports are intended to document external perspectives and do not represent official NIST positions. The opinions, recommendations, findings, and conclusions in this publication do not necessarily reflect the views or policies of NIST or the United States Government.

National Institute of Standards and Technology Special Publication 1500-17

Natl. Inst. Stand. Technol. Spec. Publ. 1500-17, 79 pages (January 2021)

CODEN: NSPUE2

This publication is available free of charge from: https://doi.org/10.6028/NIST.SP.1500-17 


\begin{abstract}
This document is the report of a workshop held in October 2019 at the National Institute of Standards and Technology (NIST) in Boulder, CO seeking input from research, industry, and regulatory communities on the photopolymer additive manufacturing (PAM) research and development agenda. The report provides a synopsis of the current state of the science and highlights challenges, needs, and future opportunities for accelerated innovation in PAM. It is intended to be used for strategic planning and decision-making purposes by PAM stakeholders and to fuel collaborations among the PAM community to accelerate innovation. The meeting was jointly hosted by NIST and RadTech, the ultraviolet and electron beam photopolymer chemistry international nonprofit organization.
\end{abstract}

\title{
Key words
}

Photopolymer Additive Manufacturing; Roadmapping Workshop; Stakeholder Collaboration.

\section{Acknowledgements}

NIST and RadTech would like to all participants of the October 29-30, 2019 Photopolymer Additive Manufacturing Workshop (PAM Workshop 2019). A complete list of attendees is provided in Appendix A. The participants braved inclement weather (freezing rain, snow, and sleet) to attend this workshop. Special thanks are extended to the members of the workshop planning committee, listed below. The committee was responsible for all essential groundwork for the event and the contents of this report.

Presentations from invited speakers and panel discussions at the workshop provided the foundation for this report. Without the enthusiastic participation of all workshop attendees, this report would not have been possible. Many thanks are also due to the conference support staff of NIST, especially Gladys Arrisueño, Terri Viezbicke, Benjamin Jeanette, and Pauline Truong, who ensured the meeting logistics were handled with excellence and efficiency. Special thanks are also due to our tour sponsors who opened their doors for attendees to visit their hands-on laboratory and industrial settings at NIST (Jim Burrus), Colorado Photopolymer Solutions (Neil Cramer and Mike Idacavage) and 3D Systems Health Care (Taylor Broaddus). Lastly, many thanks to our panel members, panel moderators and volunteer rapporteurs who are listed on the title pages of each of the workshop panel sections.

\section{Organizing Committee}

Callie Higgins, NIST, Chair

Jason Killgore, NIST, Chair

Dianne Poster, NIST

Gary Cohen, RadTech

Neil Cramer, Colorado Photopolymer Solutions

Mickey Fortune Jr., RadTech

Mike Idacavage, Colorado Photopolymer Solutions, RadTech

Cameron Miller, NIST

For more information on current NIST PAM research click here. 


\section{Table of Contents}

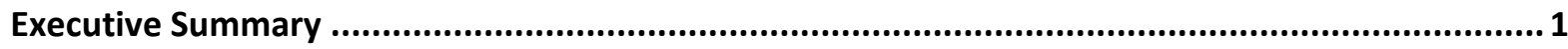

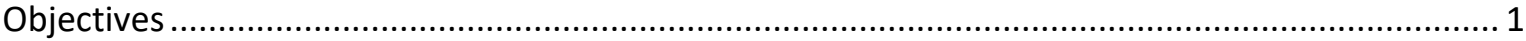

Overarching Challenges to Advancing PAM Technology............................................................

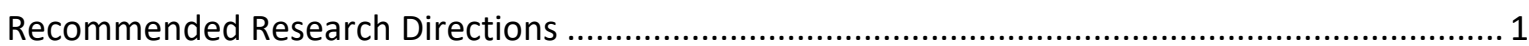

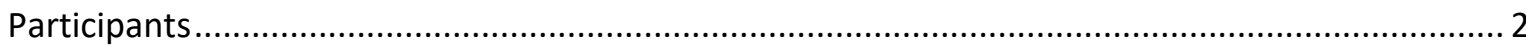

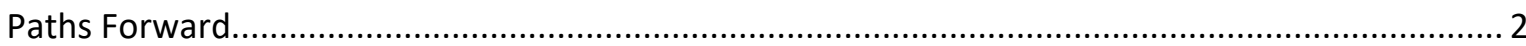

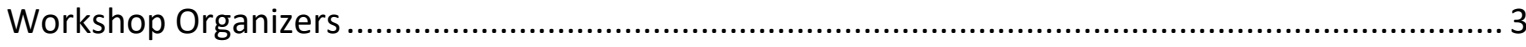

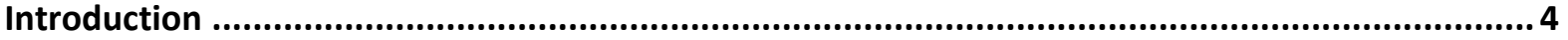

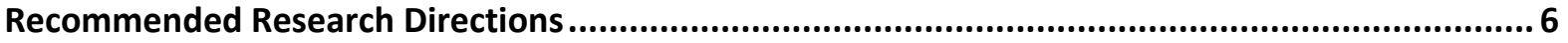

Material Science and Engineering to Characterize PAM Materials............................................ 6

Advanced Computing for Materials Discovery and Process modelling......................................... 6

Measurement Science and Standards Development to Optimize PAM .......................................... 7

Environmental, Health, and Safety Regulations for Effective PAM Adoption................................ 8

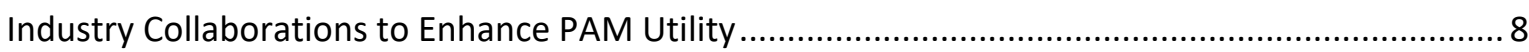

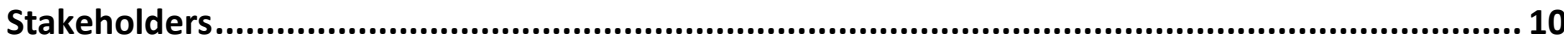

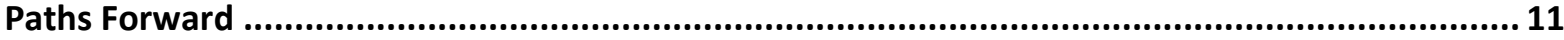

Panels

Panel 1: Sustainable, Hybrid, and Emerging Material Systems .................................................. 13

Panel II: Novel AM-Specific Characterization ............................................................................ 19

Panel III: Industry Applications and Developing Markets ............................................................. 25

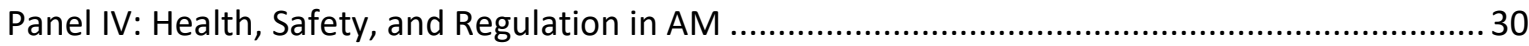

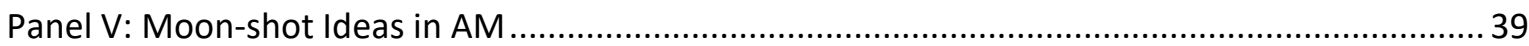

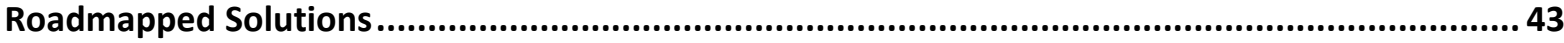

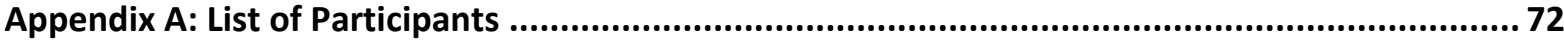

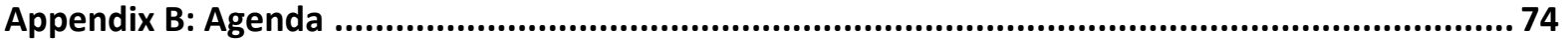




\section{Executive Summary}

\section{Objectives}

The Photopolymer Additive Manufacturing (PAM) Workshop, held October 29-30, 2019 at the National Institute of Standards and Technology in Boulder, Colorado, was organized to identify common problems and solutions specific to photopolymers, ultraviolet curing, manufacturing processes, and performance of materials in PAM commercial products. The workshop's technical focus was on the design, synthesis, and production of PAM printed products, particularly with respect to setting standards and establishing measurement science needs. The workshop comprised of five workshop panel topics, which are listed to the right.

\section{Overarching Challenges to Advancing PAM Technology}

The Photopolymer Additive Manufacturing (PAM) Workshop held at NIST in October 2019 identified four overarching challenges to advancing PAM technology:

1. Limits in materials and chemistry

2. Lack of advanced characterization and standards

3. Rapidly evolving and dynamic hardware, software, and scalability needs

4. Lack of clarity and specificity in environmental, health, and safety regulations

\section{Recommended Research Directions}

The workshop participants identified these potential pathways to progress with the corresponding roadmapped solutions in Figure 1:

I. Material science and engineering to characterize PAM materials, including in-situ and multimode studies of the PAM process (e.g., simultaneous modulus and crosslinking density measurement)

II. Advanced computing for materials discovery and process modelling of PAM parts to accurately predict performance of photopolymers on multiple length- and time-scales

III. Measurement science and standards development for reliable, high-performance PAM to overcome throughput and quality barriers, such as varying light intensity and exposure dose combined with increasing build volume capacity

IV. Environmental, health, and safety regulations for safe and responsible PAM adoption in coordination with industry and regulatory institutions

V. Industry collaborations to enhance PAM utility, such as shared test facilities for pilot programs and computing capabilities 


\section{Figure 1: Roadmapped Solutions for Advancing Photopolymer Additive Manufacturing (PAM)}

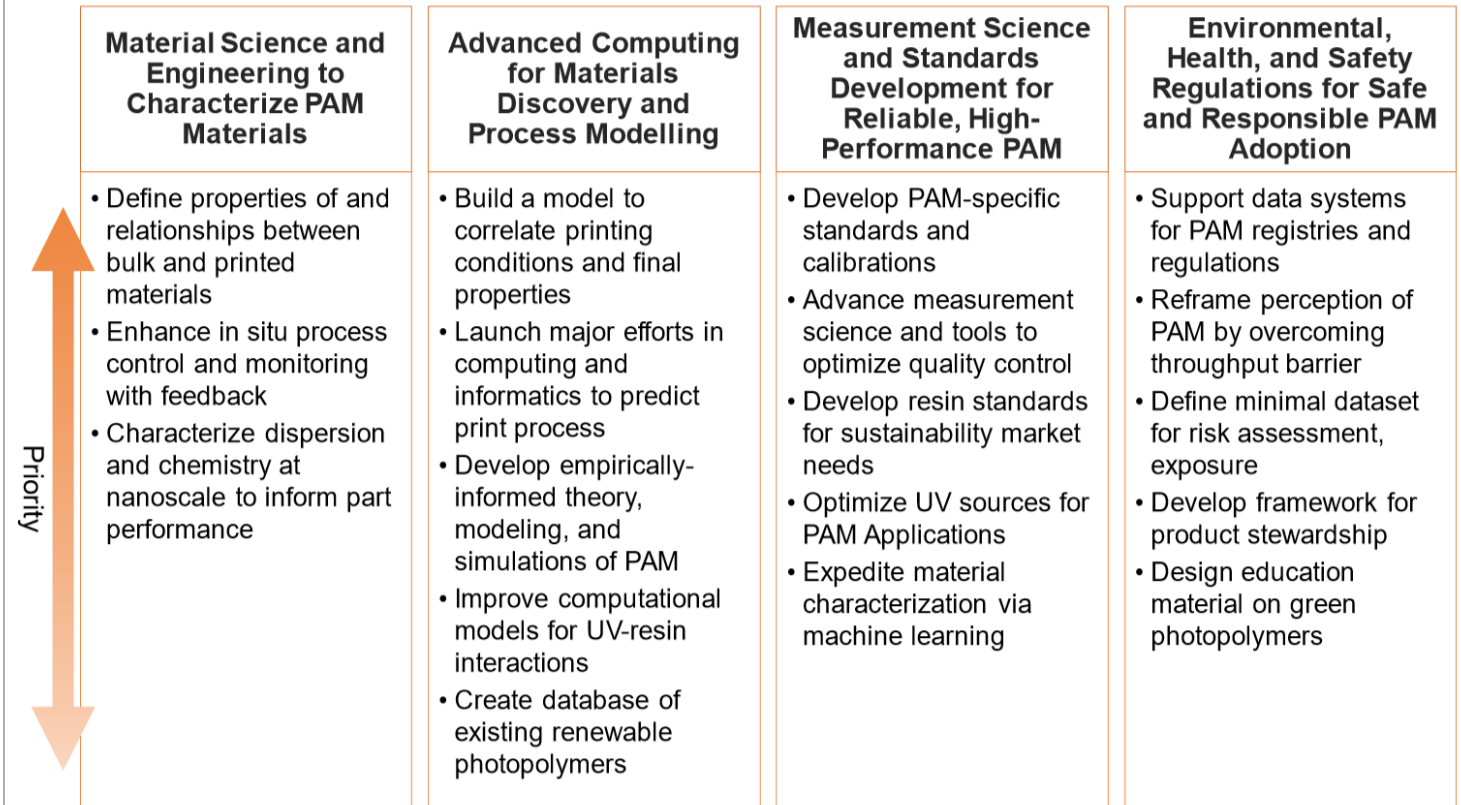

Recommended Research Directions

\section{Participants}

The PAM workshop was sponsored by the NIST Material

Measurement Laboratory and organized in collaboration with

RadTech North America, an ultraviolet and electron beam (UV+EB)

photopolymer chemistry international nonprofit organization. The

workshop drew more than 80 participants spanning all sectors of

the PAM community. The pie chart to the left provides a

breakdown of attendees per stakeholder group and Figure 2

illustrates a breakdown of stakeholder priorities as they relate to the PAM process flow.

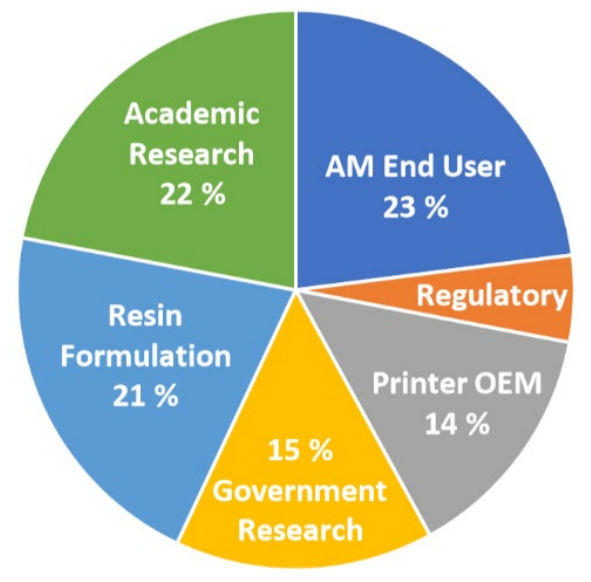

\section{Paths Forward}

1. Host webinar on workshop output and update community on current PAM challenges and opportunities

2. Establish environmental, health, and safety data to support educational outreach and regulation development

3. Convene annual meetings for ongoing engagement between industry, regulatory and research institution stakeholders

4. Institute flexible government and academic research programs to address stakeholder needs 
Figure 2: Photopolymer Additive Manufacturing (PAM) Process Flow and Priorities

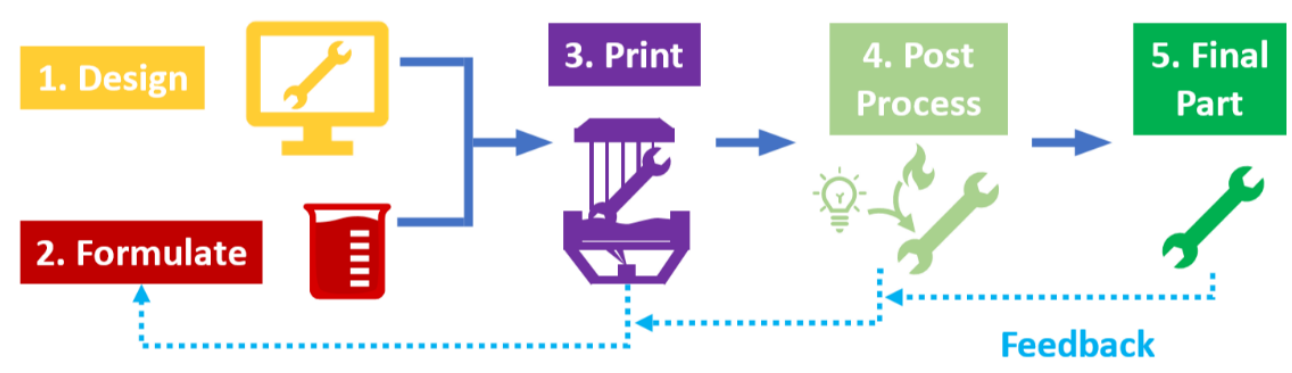

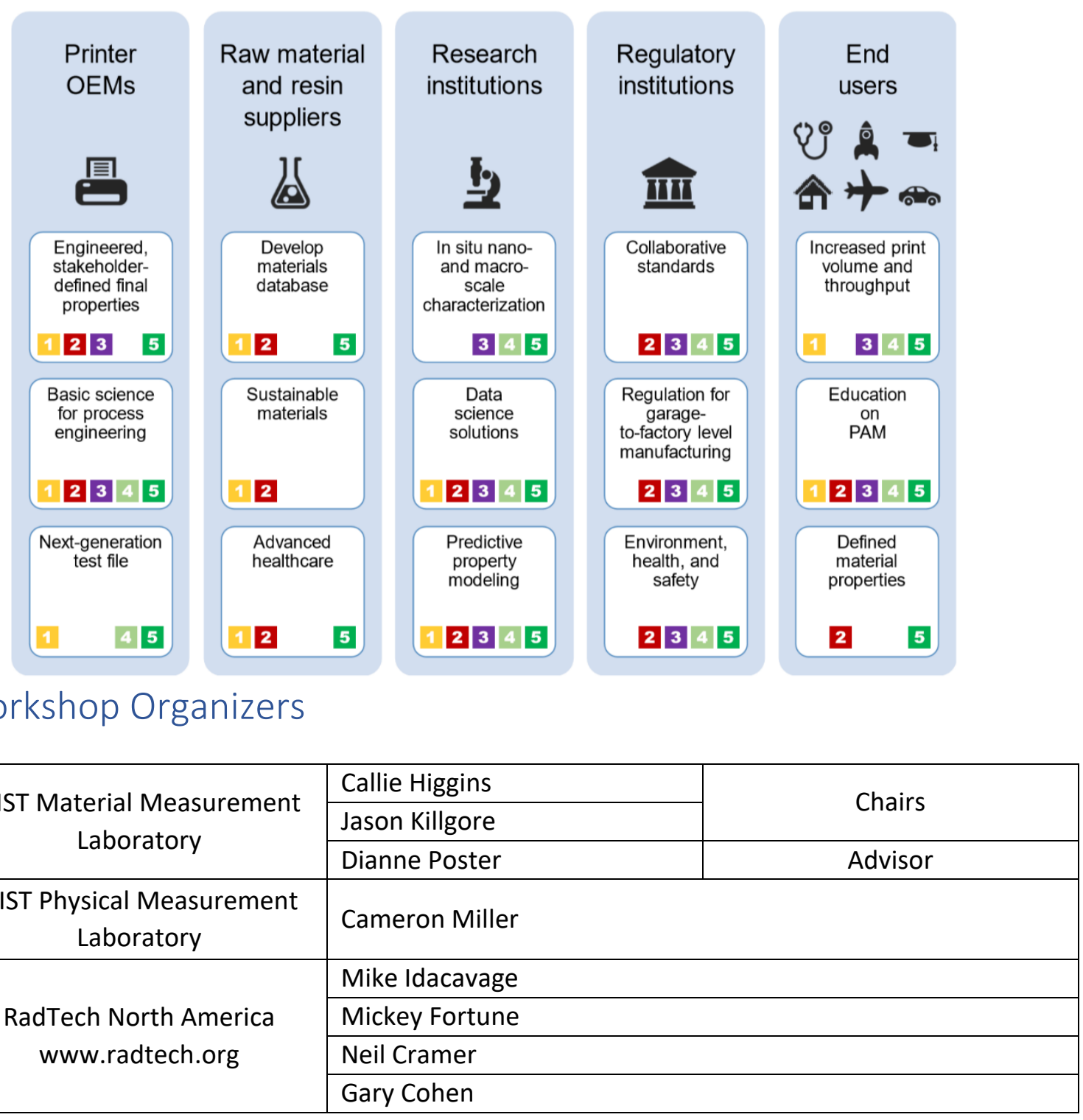




\section{Introduction}

Photopolymer Additive Manufacturing (PAM) was introduced by Charles W. Hull in 1984 via stereolithography (SLA), a technique which makes use of ultraviolet light (UV) and a bed or vat or photopolymer resin to produce 3D objects. The method produces solid objects by successively patterning 2D slices of a 3D object into thin layers of the photocurable material layer-by-layer until the entire object is formed. The UV patterning process photopolymerizes a given resin into the desired solid, 3D structure. ${ }^{1}$

In today's 3D printing market, SLA and is one of many polymer additive manufacturing technologies such as fused deposition modeling (FDM), selective laser sintering (SLS), polyjet/multijet printing, and digital light processing. PAM, which encompasses all photopolymer additive technologies, comprises the largest market share of all polymer AM technologies, and has garnered much attention from a variety of fields including healthcare, rapid prototyping, and manufacturing to name a few.$^{2}$

In March 2020, Globe Newswire released a review of ResearchAndMarkets.com market report titled "Polymer Additive Manufacturing Markets and Applications: 2020-2029". The review noted the PAM global economic impact is driven by massive opportunities in direct AM production and as such, it is anticipated polymer 3D printing may generate as much as $\$ 11.7$ billion in 2020 including sales generated by polymer AM hardware and all associated material families. Moreover, in the last two years, industrialization of PAM is described as moving forward through advanced technology. For example, end-to-end workflow automation and optimization is increasingly prevalent in the industry. Some of the most significant progress and accomplishments in PAM markets to date have been registered in material science and development/optimization of new materials for current technologies. ${ }^{3}$ However, many challenges remain for the industry for accessing new markets and application spaces, largely due to a lack of standardized tests, materials (artifacts) and measurements for quality control processes.

Robust and viable methods are needed to scale up the production of promising new materials to achieve these market predictions. Approaches to scale the industry to commercial-level production without compromising the material properties or functionalities are necessary. Fundamental questions across disciplines such as in materials science and engineering, physics, chemistry, and biology must be resolved to harness the potential benefits of new and game-changing PAM products. A cross-industry approach with multi-stakeholder engagement is necessary to solve these fundamental challenges as the many issues currently hindering widespread PAM consumer product and industrial applications are shared by all. A focused effort by industry, government and others to

\footnotetext{
${ }^{1}$ Charles W. Hull. "Method for production of three-dimensional bodies by stereolithography. Patent number: 5174943 , Issue date: December 29, 1992.

2 2019: "3D Printing Market by Offering (Printer, Material, Software, Service), Process (Binder Jetting, Direct Energy Deposition, Material Extrusion, Material Jetting, Powder Bed Fusion), Application, Vertical, Technology, and Geography - Global Forecast to 2024", October 23, MarketsAndMarkets.com

${ }^{3}$ GlobeNewswire, March 9, 2020 (Webpage)
} 
fuel innovation and address fundamental science, technology, standards, and data issues will expedite the pace of PAM advancement and benefit all stakeholders.

The National Institute of Standards and Technology (NIST) and RadTech, hosted the first Photopolymer Additive Manufacturing Workshop (PAM) 2019, in Boulder, Colorado. 3D Printing Industry representatives were among the over 80 attendees present from October 29 through 30, who fought through the sudden snowstorm to discuss the topic: "Roadmapping a Future for Stereolithography, Inkjet, and Beyond."

More specifically, PAM 2019 consisted of 21\% resin suppliers, 14\% OEMs, 22\% academic institute members, 15\% representing Government Research Agencies, 5\% Government Regulatory Agencies, and 23\% end-users. In the PAM 2019 opening remarks, Dr. Frank Gayle, Deputy Director of the Office of Advanced Manufacturing at NIST stated:

"70 million jobs have been made obsolete due to automation and new technologies. However, 130 million jobs have been birthed as a result of additive manufacturing. Addressing the skills gap as well as other limitations of PAM will only prosper the burgeoning technology which is seen in industries such as defense and energy, automotive, and healthcare."

It is clear given the current global 3D printing market, small and large companies must find approaches that are affordable and technologically feasible to address these limitations. Companies must seek ways to partner collectively with government and academic stakeholders to enable PAM processes and products to add value, improve product performance, and open new markets to provide competitive advantage over other manufacturing technologies. 


\section{Recommended Research Directions}

The 23 specific roadmapped solutions developed by the workshop attendees fall into five Recommend Research Directions (RRDs). Here, these five Recommend Research Directions are listed along with the corresponding roadmapped solutions grouped by high, medium, and lower priority levels as determined by PAM Workshop attendees. A few key points are provided here for each solution. The Roadmapped Solutions Chapter later dives deeply into each roadmapped solution.

\section{Material Science and Engineering to Characterize PAM Materials}

In order to compete with traditional polymer processing and competitive AM technologies, PAM must produce parts with reliable and high performing material properties. Efforts that investigate materials at multiple process stages, including in situ, are needed to understand why PAM parts mechanically underperform their bulk counterparts and enable a broader set of materials for PAM.

High Priority Roadmapped Solutions:

Define properties of and relationships between bulk and printed materials

- $\quad$ Probe the relationship between interlayer adhesion and final print strength to optimize final print properties

- Study dynamics of printing material in various forms: resin, pre-gelation, gelation, fully converted, and post processed

Medium Priority Roadmapped Solutions:

Enhance in situ process control and monitoring with feedback

- Mature characterization technologies to understand material properties in the native printing environment which govern print resolution and minimum feature size

- Monitor mechanical and chemical properties during the printing process to inform feedback mechanisms to optimize final print

Lower Priority Roadmapped Solutions:

Characterize dispersion and chemistry at nanoscale to inform part performance

- Probe the relationship between dispersion of resin components and final part performance

- Optimize filler dispersion and compatibility between fillers and polymer matrices to develop high performance parts

\section{Advanced Computing for Materials Discovery and Process modelling}

As a digital manufacturing technology, PAM must leverage advanced computational methods to discover new materials and ensure their optimal processability. Computational methods that include physics-based models, data-driven models, and combinations thereof can be used to simultaneously optimize materials, processing, and design and to enable the development of new materials and processes. The development of robust databases is essential to inform and validate computational approaches.

High Priority Roadmapped Solutions:

Build a model to correlate printing conditions and final properties

- Final print properties determine utility of PAM technology and a model of printing process to inform ways to increase throughput while maintaining print quality

Launch major efforts in computing and informatics to predict print process 
- Harness large amounts of data generated by printing process to characterize and optimize PAM for a given resin and printing condition facilitated by advances in computing capacity

Medium Priority Roadmapped Solutions:

Develop empirically-informed theory, modeling, and simulations of PAM

- To fully engineer the functionality of PAM parts and increase print throughput, comprehensive theory, modeling, and simulations of the printing process is required

Improve computational models for UV-resin interactions

- Models informing UV-resin interactions will facilitate increased build efficiency

Lower Priority Roadmapped Solutions:

\section{Create database of existing renewable photopolymers}

- Develop knowledge hubs on current understanding of reinforcements and additives to expedite high performance, renewable materials for PAM

- Create (or centralize existing) toxicity databases will decrease confusion regarding safety protocols and expedite PAM adoption

\section{Measurement Science and Standards Development to Optimize PAM}

The reliance on proprietary materials, testing and componentry limits fundamental scientific insight and the sharing of knowledge, techniques, and best practices between stakeholders. There must be standardized test geometries, resins, and light sources for development purposes. Test methods should leverage the process control inherent to PAM to build parametric datasets. New material classes, such as sustainable (e.g. bio-based, recyclable) resins can particularly benefit from standards.

High Priority Roadmapped Solutions:

Develop PAM-specific standards and calibrations

- Standards and calibrations development for both PAM resins and the PAM process to expedite the optimization of printed parts while making technology more accessible to new applications

\section{Advance measurement science and tools to optimize quality control}

- Optimize PAM part quality for given applications and invest in versatile instruments to probe a variety of resins and print conditions

Medium Priority Roadmapped Solutions:

Develop resin standards for sustainability market needs

- Define and implement sustainable resin standards for successful adoption

Optimize UV sources for PAM Applications

- Determine the most effective and efficient photopolymerization sources critical to process control

Lower Priority Roadmapped Solutions:

Expedite material characterization via machine learning

- Use data generated by experimental PAM to inform machine learning algorithms designed to characterize a given material 


\section{Environmental, Health, and Safety Regulations for Effective PAM Adoption}

PAM technology adoption has spread rapidly in recent years, catching regulatory agencies by surprise. Given the unique handling requirements of PAM resins and post-process needs of PAM parts, specific data, guidelines and education for user and environmental safety are needed.

High Priority Roadmapped Solutions:

\section{Support data systems for PAM registries and regulations}

- Engage proactively with regulators and legislators in setting standards for new materials registrations

- Industry associations inform and engage with safety and regulations continuously and provide information sessions at meetings, workshops etc.

Reframe perception of PAM by overcoming throughput barrier

- Create awareness for utilization of PAM in industries requiring customization while increasing throughput by optimizing and regulating the print process

Medium Priority Roadmapped Solutions:

Define minimal dataset for risk assessment, exposure

- Adopt approaches and methods from other industries, such as nanotechnology, or develop new methods for streamlining risk assessment

\section{Develop framework for product stewardship}

- Build safety concepts into product launch models and business plans

- Collect, review, and disseminate current and best practices to enhance life cycle analysis

- Integrate circular economy frameworks into PAM processes and materials

Lower Priority Roadmapped Solutions:

Design education material on green photopolymers

- Facilitate adoption of green photopolymers by communicating with stakeholders the utility of sustainable materials for use in PAM

\section{Industry Collaborations to Enhance PAM Utility}

Many science and engineering challenges must be addressed for PAM to achieve its full potential. The rapid pace of innovation by PAM industry requires alignment amongst stakeholders to ensure priority research needs are being addressed. Fundamental and applied research investments must be balanced to ensure short-term and long-term successes.

High Priority Roadmapped Solutions:

Translate commercial needs into R\&D efforts

- Improve industry coordination in communicating research needs to research community

- Create communication structure between academia, government, and industry to enable pre-competitive research sharing

Support collaborations between small- and large-scale PAM industry adopters

- Develop models for intellectual property protection and licensing between private and public sectors

- Improve technology transfer to match PAM capabilities to industry needs

- Entrepreneurship and technology transfer training into academic programs to support small PAM manufacturing companies 
Medium Priority Roadmapped Solutions:

Advance manufacturing capabilities in PAM

- Create programs to sponsor facilities for testing and shared use production for PAM implementation in novel markets

- Use such facilities to expedite PAM resin formulation, resin testing, and pilot programs Build value proposition to meet customer needs

- Clearly define utility of PAM for a variety of application spaces

- Prioritize commercial needs and translate these areas of study into research funding investments

Lower Priority Roadmapped Solutions:

Pursue precision engineering and manufacturing

- Highlight PAM's unique capacity to realize precision engineering and manufacturing via versatile materials and print processes

- Create programs to elevate data science value among PAM manufacturers 


\section{Stakeholders}

The workshop aimed to gather all relevant photopolymer AM stakeholders to develop a roadmap to drive innovation throughout the field. Stakeholders were broken down into six distinct groups, each representing critical areas of expertise and perspective.

AM End user workshop attendees represented a wide variety of industries with a vested interest in the advancement of this technology including the automotive industry, medical device companies, and other major technology companies. This group represents the growing demand for PAM across industry sectors where customization and rapid deployment are of the utmost importance.

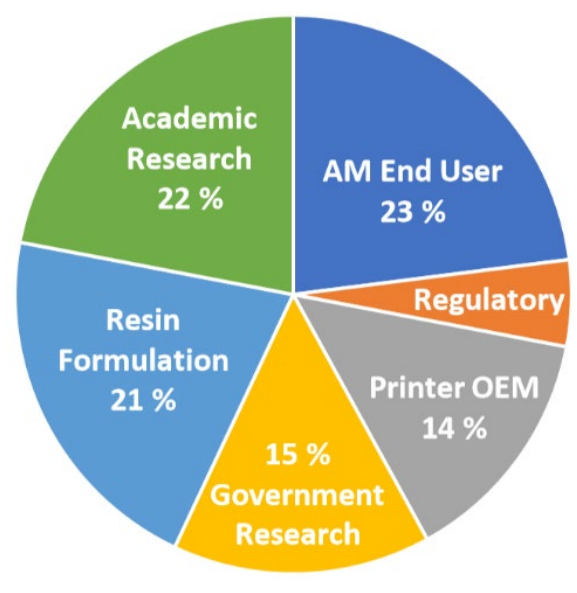

Resin formulators included representatives from specialized resin formulators, major chemical manufacturers, and PAM-specific resin suppliers. This group highlights the presence of active investment into the advancement of this technique as a manufacturing technology and the innovative materials that are unique to PAM. Resin manufacturers work closely with all stakeholder groups to inform material development for the diverse range of PAM applications.

Printer OEMs represented nearly every major photopolymer AM printing method on the market including digital light synthesis, stereolithography, digital light processing, and high area rapid printing. The rapidly evolving nature of this stakeholder group indicates the need for collaborative relationships to drive innovation throughout the industry while providing the driving mechanism for growth in PAM.

Academic researchers represented research efforts ranging from major additive manufacturing research centers to single research groups. This stakeholder group includes PAM research efforts in material characterization (resin and final part), personalized medicine, and machine learning. Connecting this group to other stakeholders is essential to the acceleration of PAM into manufacturing due to the creativity afforded and fostered at academic institutions.

Regulatory institutes highlighted the need for a greater regulatory and standards presence throughout the industry. This stakeholder group included representatives from the areas of highest regulation demand in PAM including consumer protection, environmental protection, and occupational safety. Regulatory institutes in this group presented the need to understand the entire workflow of PAM from the use of resins in the printer to the post-processing of final parts.

Government researchers made up $15 \%$ of the PAM workshop attendance and represented the government's investment in driving innovation in the United States through PAM. This group included laboratories from the Department of Energy, Department of Defense, and the Department of Commerce, indicating the versatile nature of PAM across disciplines and applications. These stakeholders provide guidance from a unique vantage point that can serve as a pre-competitive driving force behind innovation across all stakeholder groups. 


\section{Paths Forward}

As the RRDs of this workshop illustrate, there are numerous cross-industry challenges related to PAM that could begin to be addressed through a concerted, integrated public-private effort. The following paths forward serve as a foundation for a coordinated effort across stakeholder groups:

1. Host webinar on workshop output and update community on current PAM challenges and opportunities

2. Establish environmental, health, and safety (EHS) data to support educational outreach and regulation development

3. Convene annual meetings for ongoing engagement between industry, regulatory and research institution stakeholders

4. Institute flexible government and academic research programs to address stakeholder needs Identifying the cross-industry challenges to PAM is essential to develop a foundation for cost-effective mainstream PAM. As PAM databases emerge regarding types of materials, processes, and EHS, the need for a strong consensus for test methods will become greater. Innovations in raw materials, new UV curing technologies, formulations, equipment and evaluation of PAM products will all be important.

While there is some federal investment in PAM research, the challenge remains in prioritizing what needs to be done academically verses commercially, and how to leverage the outcomes (gaining the most benefit for dollars spent). Regulatory agencies are beginning to make a push toward crossindustry programs to expand EHS R\&D. RadTech has pledged to commit resources to develop the necessary data and information required by regulators and others that may have health and safety concerns about PAM. This is a concrete example of a group inspired by PAM 2019 to continue the ideas and efforts discussed.

The need for coordination in manufacturing R\&D and information dissemination across the public and private sector has never been stronger and is critical to attaining and maintaining global leadership for American companies. This coordination will be essential to open markets and expand PAM through innovation. Implementing a multi-stakeholder framework will require the development of coordinating entities with members from the private and public sectors. A first next step should be a follow-up meeting to PAM 2019 with the clear goal to identify and explore mechanisms and frameworks for collaborating as an industry with the public going forward. A pre-conference workshop or a multi-day session at a future RadTech meeting could be this next step. 


\section{Panels}

The workshop was structured to identify common problems and solutions specific to PAM such as feedstock materials, manufacturing processes, and performance of printed products within widely different industries including the chemical, biomedical, and materials manufacturing sectors with representatives from the 3D printer manufacturers, resin suppliers, and AM end-users. Panel sessions provided a venue for participants to focus on the topics identified as they apply to the design, synthesis, and production of PAM products. The panel session topics are identified in the table below.

\section{Panels for PAM 2019, Boulder, Colorado, October 29 - 30, 2019}

I. Sustainable, hybrid, and emerging material systems

II. Novel, AM-specific characterization

III. Industry applications and developing markets

IV. Health, safety and regulation in AM

V. Moon-shot ideas in AM

Within each of these topics, common issues discussed included materials, manufacturing, measurement, characterization, modeling, high-performance properties, and environmental, health and safety concerns. Throughout the workshop, emphasis was placed on identifying the needs that were cross-cutting in nature and could impact multiple industries and products for PAM. 


\section{Panel 1: Sustainable, Hybrid, and Emerging Material Systems}

Moderator: Mike Idacavage, RadTech

Rapporteur: Jason Killgore, NIST

Panelists: Eric Romano, DSM

Jeff Klang, Sartomer

Alex Mejiritski, Spectra Group Limited

Rachel Davis, Azul3D

Justin Poelma, Carbon

Neil Cramer, Colorado Photopolymer Solutions
Panel Scope

- Identify fundamental metrology and chemistry needs for material systems at scales ranging from nano to macro

- Discuss and refine needs to address fundamental science and education gaps with respect to developing industryappropriate and sustainable materials

- Consider ways to enable scale-up of PAM

One of the keys to advancing PAM is building the materials science and technology base to enable new pathways to product specialization, differentiation, and customization. New material systems that can be designed based on existing and emerging science are needed. These systems must be: 1 ) capable of being easily and routinely inserted into equipment or systems that are in use today; 2 ) designed to retain and combine their unique properties in a system of other materials; and 3) able to provide superior and controllable performance relative to traditional systems.

\begin{tabular}{|c|c|c|}
\hline \multicolumn{3}{|c|}{ Basic Materials in PAM } \\
\hline Process & Attributes & Effects \\
\hline \multicolumn{3}{|c|}{ Acrylic- and Methacrylic-based Resins } \\
\hline $\begin{array}{l}\text { Free radical } \\
\text { polymerization }\end{array}$ & $\begin{array}{l}\text { - Wide range of } \\
\text { functionalities } \\
\text { - High or Low } \\
\text { molecular } \\
\text { weight (MW) }\end{array}$ & $\begin{array}{l}\text { - Changing functionality of resin components allows } \\
\text { for control of photopolymerization speed, } \\
\text { heterogeneity, and final part properties } \\
\text { Lower MW affords higher reactivity and stiffness, } \\
\text { while higher MW offer flexibility and reaction } \\
\text { stability }\end{array}$ \\
\hline \multicolumn{3}{|c|}{ Epoxy or Vinyl Ether-based Resins (same as above plus the following) } \\
\hline $\begin{array}{l}\text { Free radical and } \\
\text { cationic } \\
\text { polymerization }\end{array}$ & $\begin{array}{ll}\text { - } & \text { Dilution } \\
& \text { power } \\
\text { - } & \text { Reduced } \\
& \text { toxicity }\end{array}$ & $\begin{array}{l}\text { - Tunable photopolymerization rate } \\
\text { - Larger chain structure, higher functionality and } \\
\text { more voluminous groups lead to greater viscosity } \\
\text { - Epoxides specifically provide higher mechanical } \\
\text { properties, lower shrinkage, and better chemical } \\
\text { and thermal resistance. }\end{array}$ \\
\hline
\end{tabular}

Historically, the first photocurable materials used in SLA applications consisted of a urethane dimethacrylate with a small fraction of acrylic acid, benzophenone as photoinitiator, and methyl ethyl hydroquinone/triallyl phosphate to inhibit premature polymerization. 4 Over time, various photocurable materials have been developed using free radical and ionic polymerization (see table above). Free radical approaches are more commercially in use today, with acrylates widely employed; however, ionic systems are finding increasing applications. Materials are selected for a processing technology and application based upon a variety of resin properties including viscosity,

\footnotetext{
${ }^{4}$ Hull, C. W.; Lewis, C. W. U.S. Patent 4999143, 1991.
} 
curing speed, cost, shelf life, volatility, and toxicity that are achieved either by a single material or a system of materials used in combination.

Different combinations of monomers, oligomers, photoinitiators, and various other additives result in products with a variety of mechanical and chemical properties. Mechanical properties and performance attributes are especially important, including toughness, durability, heat resistance, and strength. Currently, a large portion of 3D printed polymer products are used as conceptual prototypes rather than as functional components because current pure photopolymer products lack strength and application versatility. Design of materials with engineered structural or functional properties that make use of particle, fiber or nanomaterial additives provide opportunities to enhance strength and function of printed objects. For example, the blending of a thermoelectric material, such as $\mathrm{Bi}_{0.5} \mathrm{Sb}_{1.5} \mathrm{Te}_{3}$ (BST) into SLA resins can result in composites with ultralow thermal conductivity, which is favorable for thermoelectric applications in aerospace. The ability to fabricate reinforced polymer matrix composites is anticipated to boost PAM applications across multiple industries beyond conceptual prototyping and into larger scale systems. ${ }^{5}$

\begin{tabular}{|l|l|l|}
\hline \multicolumn{1}{|c|}{ Example Composite Materials for PAM } \\
\hline \multicolumn{1}{|c|}{ Reinforcement } & \multicolumn{1}{|c|}{ Materials } & \multicolumn{1}{c|}{ Effects } \\
\hline $\begin{array}{l}\text { Particle reinforced } \\
\text { polymer } \\
\text { composites }\end{array}$ & $\begin{array}{l}40 \% \text { aluminum } \\
\text { oxide }\left(\mathrm{Al}_{2} \mathrm{O}_{3}\right)\end{array}$ & $\begin{array}{l}\text { - } \\
\text { Improved dielectric permittivity }\end{array}$ \\
\hline Nanocomposites & $\begin{array}{l}\text { Reduced dielectric loss tangent } \\
\text { carbon nanotubes, } \\
\text { fiber, } \mathrm{TiO}_{2}, \mathrm{BaTiO}_{3}, \\
\mathrm{Bi}_{0.5} \mathrm{Sb}_{1.5} \mathrm{Te}_{3}\end{array}$ & $\begin{array}{l}\text { Improved tensile strength and modulus, flexural } \\
\text { strength, hardness, thermal stability, } \\
\text { piezoelectric coefficient, and electromagnetic } \\
\text { absorption } \\
\text { Ultralow thermal conductivity and high energy } \\
\text { conversion efficiency }\end{array}$ \\
\hline
\end{tabular}

Sustainable, hybrid and emerging material composites will enhance the versatility of PAM applications by offering advantages over traditionally manufactured parts such as light weight, high mechanical strength, high performance electrical properties, superior reliability in extreme environments, and even unique color or texture capabilities. Applications could be as diverse as biological implant materials, electronic packages, and automotive or aircraft components.

Additives to polymers can enable new properties in composites, however the resulting performance of PAM products will be highly dependent on the processing used. For example, often non-reactive additives or "...hybrid material systems will be gaining interest as that greatly expands the performance of an AM printed part for commercial applications".

Mike Idacavage, RadTech solvents are used to decrease the - August 29, 2019, 3D Printing Industry viscosity of photopolymer resins. Understanding how these additives

\footnotetext{
${ }^{5}$ Xin Wang, Man Jiang, Zuowan Zhou, Jihua Gou, David Hui, 3D printing of polymer matrix composites: A review and prospective, Composites Part B: Engineering, Volume 110, 2017, Pages 442-458, https://doi.org/10.1016/i.compositesb.2016.11.034.
} 
affect processing parameters and final product performance is essential. Industry is seeking the development of new formulations specific for 3D, rather than continue adopting formulations originally designed for 2D. Currently, there is no set of rules or guide available for mixing multiple components to obtain the required properties in a composite structure. Research infrastructure supporting the development of critical data to better understand, model and predict emerging material systems will be essential.

Considerations on the degradability and recycling of the final products and other environmental aspects, such as life cycle analysis, sustainability, and green chemistry approaches, are only just beginning to be incorporated into the PAM supply chain and are perhaps the next generation of PAM. Education, training, and public engagement on these topics are equally important.

Advances in equipment will be critical to expand the applications of this technology, particularly at larger scale. For example, emerging formulations that can be photopolymerized under longer wavelengths to achieve higher penetration depth may be important, requiring advancements in UV and other light sources. In general, innovation will involve new material formulations combined with advances in processing/equipment and will require multidisciplinary collaborative research efforts.

\section{Current State and Technological Advances}

Liquid photopolymers used in PAM are generally acrylate-based or epoxy-based photocurable resins. High printing resolution is obtained via laser scanning and UV-induced curing processes. Resolution in the $Z$ direction is at the 10 micrometer scale with printers produced for today's market (for example, DWS Systems), making PAM an attractive alternative to traditional polymer-based AM techniques which have lower resolution. The higher resolution offered by PAM enables production of parts with complex geometries, dimensional accuracy and precision, and low levels of surface roughness. ${ }^{6}$

More polymers are being synthesized containing aliphatic polyesters which allow for biodegradation, and in some cases allow for the encapsulation of cells. Soft materials are receiving attention as well, such as hydrogels ${ }^{7}$. These advances have opened doors for PAM applications in tissue engineering, such as cartilage or bone tissue engineering, in addition to the already burgeoning applications of PAM in the medical fields. ${ }^{8}$

\footnotetext{
${ }^{6}$ Shalza Sharma, Avinash Chauhan, Andriya Narasimhulu, 2019, A Review of Recent Developments on Stereolithography, International Journal of Engineering Research \& Technology (IJERT) Volume 08, Issue 08 (August 2019)

${ }^{7}$ Callie I. Higgins, Jason P. Killgore, Frank W. DelRio, Stephanie J. Bryant, and Robert R. McLeod, Photo-tunable hydrogel mechanical heterogeneity informed by predictive transport kinetics model https://doi.org/10.1039/D0SM00052C Soft Matter, Advance Article 2020

${ }^{8}$ Chia, Helena N, and Benjamin M Wu. "Recent advances in 3D printing of biomaterials." Journal of biological engineering vol. 94.1 Mar. 2015, https://dx.doi.org/10.1186\%2Fs13036-015-0001-4 .
} 


\begin{tabular}{|c|c|c|c|c|}
\hline \multicolumn{5}{|c|}{ Photopolymer Printing Techniques } \\
\hline Technique & Starting Material & Example Polymers & Approach & $\begin{array}{l}\text { Resolution } \\
(\mu \mathrm{m})\end{array}$ \\
\hline $\begin{array}{l}\text { Stereo- } \\
\text { lithography (SLA) }\end{array}$ & $\begin{array}{l}\text { liquid photo-polymer } \\
\text { resins }\end{array}$ & $\begin{array}{l}\text { acylate based or } \\
\text { epoxy resins }\end{array}$ & $\begin{array}{l}\text { laser scanning } \\
\text { polymerization }\end{array}$ & $<10$ \\
\hline $\begin{array}{l}\text { Digital light } \\
\text { processing (DLP) }\end{array}$ & $\begin{array}{l}\text { liquid photo-polymer } \\
\text { resins }\end{array}$ & $\begin{array}{l}\text { various, including } \\
\text { particle- reinforced } \\
\text { resins }\end{array}$ & $\begin{array}{l}\text { selective } \\
\text { polymerization of } \\
\text { a surface by a 2D } \\
\text { projection }\end{array}$ & $\approx 10$ \\
\hline Inkjet printing & $\begin{array}{l}\text { liquid photo-polymer } \\
\text { resins, shear-thinning }\end{array}$ & $\begin{array}{l}\text { polylactic acid } \\
\text { liquid gels or } \\
\text { pastes, hydrogels }\end{array}$ & $\begin{array}{l}\text { Extrusion } \\
\text { (pressurized } \\
\text { syringe) and heat } \\
\text { or UV assisted } \\
\text { curing }\end{array}$ & $5-200$ \\
\hline $\begin{array}{l}\text { Volumetric } \\
\text { printing }\end{array}$ & $\begin{array}{l}\text { High viscosity liquid } \\
\text { or thermally gelled } \\
\text { photo-polymer resins }\end{array}$ & $\begin{array}{l}\text { gelatin } \\
\text { methacrylate, } \\
\text { acrylate resins }\end{array}$ & $\begin{array}{l}\text { Tomographic } \\
\text { printing }\end{array}$ & $\approx 300$ \\
\hline Micro-SLA & $\begin{array}{l}\text { liquid photo-polymer } \\
\text { resins }\end{array}$ & $\begin{array}{l}\text { acylate based or } \\
\text { epoxy resins }\end{array}$ & $\begin{array}{l}\text { Super-resolution } \\
\text { laser scanning } \\
\text { polymerization } \\
\text { paired with } \\
\text { inhibition }\end{array}$ & $0.1-0.5$ \\
\hline $\begin{array}{l}\text { Two-photon } \\
\text { polymerization }\end{array}$ & $\begin{array}{l}\text { liquid photo-polymer } \\
\text { resins }\end{array}$ & $\begin{array}{l}\text { acylate based or } \\
\text { epoxy resins }\end{array}$ & $\begin{array}{l}\text { Polymerization } \\
\text { requiring two } \\
\text { photons to cleave } \\
\text { photoinitiator, } \\
\text { reducing } \\
\text { polymerization } \\
\text { cross section }\end{array}$ & $\approx 0.01$ \\
\hline
\end{tabular}




\section{Overarching Challenges}

Specific Overarching Challenges limiting sustainable, hybrid, and emerging materials for PAM identified in the workshop with the associated needs to address each challenge summarized below:

\begin{tabular}{|c|}
\hline Overarching Challenges and Needs in Sustainable, Hybrid, and Emerging Materials \\
\hline Limits in materials and chemistry \\
\hline $\begin{array}{l}\text { - Formulate resins with enhanced performance beyond acrylates, methacrylates, and epoxies } \\
\text { - Enhance PAM resin properties (e.g. mechanical, optical, magnetic, thermal) via composites } \\
\text { - } \text { and additives } \\
\text { - } \quad \text { Design materials that enable faster build speed } \\
\text { - Build education programs to be distributed across academic institutions and industry } \\
\text { - Research reliable visible light photoinitiators to reduce material cost }\end{array}$ \\
\hline Lack of advanced characterization and standards \\
\hline $\begin{array}{l}\text { - Measure curing and performance of materials at various length and time scales } \\
\text { - } \quad \text { Create database of standardized resin and print properties } \\
\text { - } \text { Measure time-dependent properties of curing resins } \\
\text { - } \quad \text { Establish reference methods and materials for PAM applications }\end{array}$ \\
\hline Rapidly evolving and dynamic hardware, software, and scalability needs \\
\hline $\begin{array}{l}\text { - Move from resins originally formulated for bulk processes to those specific for 3D PAM } \\
\text { - Scale-up of resins and PAM processes for industrial use } \\
\text { - Use in-situ material measurement information to control part properties } \\
\text { - Optimize UV-resin interaction per application } \\
\text { - Ensure PAM equipment supports new, sustainable materials } \\
\text { - Assess financial risks for implementing PAM }\end{array}$ \\
\hline Lack of clarity and specificity in environmental, health, and safety regulations \\
\hline 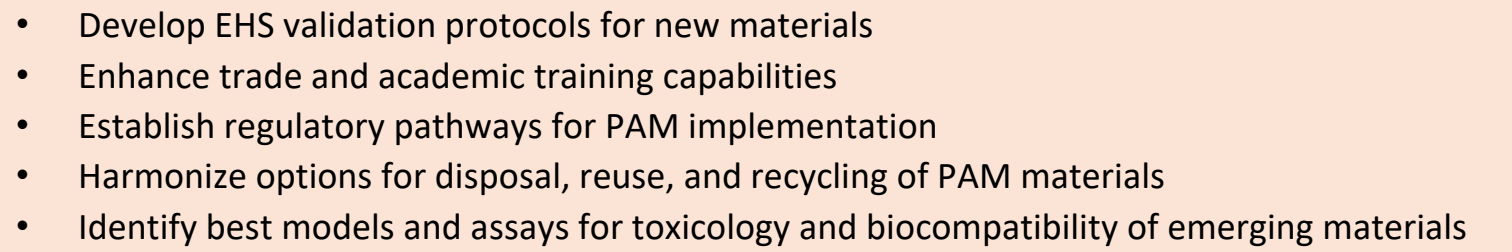 \\
\hline
\end{tabular}




\section{Recommended Research Directions}

The themes that emerged from the workshop were distilled into Recommended Research Directions with a series of solutions presented below. Bold items are included in the roadmapped solutions:

\section{Recommended Research Directions in Sustainable, Hybrid, and Emerging Materials}

Material Science and Engineering to Characterize PAM Materials

- Define properties of and relationships between bulk and printed materials

- Develop low-cost raw materials with property enhancements for accessible industry pilots

- Incorporate reinforcing components to existing materials

- Consider scale-up issues from product development and pilot outsets

- Develop approaches for composite dispersion, batch and large scale

Advanced Computing for Materials Discovery and Process modelling

- Improve computational models for UV-resin interactions

- Create database of existing renewable photopolymers

- Develop databases for PAM materials and their properties (e.g., aging, mechanical)

- Determine approaches to fund and advance multi-scale computing for PAM

- Establish tool kits to simulate multiple different properties of new materials

- Develop collaborative exchanges of property data for emerging materials

Measurement Science and Standards Development to Optimize PAM

- Develop resin standards for sustainability market needs

- Optimize UV sources for PAM applications

- Support R\&D on high-throughput methods to characterize multiple properties simultaneously

- Create programs for scale-up facilities (testing, shared use production)

Environmental, Health, and Safety Regulations for Effective PAM Adoption

- Identify chemical/physical properties for risk management decisions along material life cycle

- Develop well-characterized sample materials for testing for use by the industry

- Collect and disseminate best practices for toxicology, life cycle, and sustainability efforts

- Address third- party certification for the physical and chemical properties of PAM products

- Facilitate engagement between PAM stakeholders to set standards for new materials

\section{Industry Collaborations to Enhance PAM Utility}

- Design education materials on green photopolymers

- Build value proposition to meet customer needs

- Create and provide access to test-scale manufacturing equipment for process validation

- Develop generic templates for PAM risk assessment

- Create infrastructure capabilities for equipment, materials fabrication, testing, and pilots

- Develop models for technology transfer between public and private sector entities

- Improve industry communication of research needs to research community 


\section{Panel II: Novel AM-Specific Characterization}

Moderator: Callie Higgins, NIST

Rapporteur: Mike Idacavage, RadTech

Panelists: Jason Killgore, NIST

Max Zieringer, Formlabs

Jeff Stansbury, University of Colorado

Xiaojiang Wang, Ford Motor Company

Anthony Kotula, NIST
Panel Scope

- Identify what AM-specific characterization needs are critical to translating material interactions at the voxel-scale to end-user applications

- Define measurement and standards needs related to curing sources

- Explore ways to enable essential collaboration between research institutions and industry

The techniques and industry processes to build PAM products are the result of input streams (e.g., resin type, cure exposure conditions, layering approach) that require precise, accurate and comprehensive characterization methods to ensure the final products meet the performance needs of the end-users. Instrumentation and measurement science (metrology) are key to advancing the fundamental science and manufacturing of new PAM products. Reliable and reproducible characterizations of input and product material properties and performance are essential at the bulk-, micro- and nanoscales to ensure the emerging PM enterprise success and growth.

The characterization of raw materials, development of material properties for design, in-situ process and feedback control, workflow optimization, and modeling final properties, are essential. However, there are major challenges related to the chemistry and physics of photocuring polymers and their use inbuilding 3D parts. For example, inhomogeneous curing may cause variable mechanical properties throughout the product. These defects can lead to premature, possibly catastrophic, failure and clearly signal the critical need for materials testing standards. ${ }^{9}$ As new materials enter PAM input streams and novel, high-performing products are fabricated, assembled, and manufactured into usable parts, standardized instrumentation and measurements will be critical to providing quality control and ensuring reproducible performance across and between the PAM industries.

Looking ahead on the horizon the PAM community and its stakeholders envision new PAMbased industries that will be mass producing PAM products. Technologically advanced, i.e., smart, materials, will likely be part of the industry growth. High-performance and cost-effective instrumentation and multi-scale measurement methods will be required, along with effective collection, transmission, and interpretation of data. As more data develop, there will be substantial need for investments in expertise and infrastructure for data curation and data analytics with advanced tools to extract data, make predictions and discover trends. Tools to visualize patterns and trends, as well as machine learning technologies, will be necessary to draw meaningful conclusions from complex and varied data sources. Access to affordable computation power will be essential to consider in the future.

\footnotetext{
9 2015: NISTIR 8059, Materials Testing Standards for Additive Manufacturing of Polymer Materials: State of the Art and Standards Applicability, Aaron M. Forster, http://dx.doi.org/10.6028/NIST.IR.8059
} 
Educational programs supporting fundamental photochemistry and polymer chemistry and physics are needed to create a multi-disciplinary community that is skilled in measurements, data and instrumentation use across length and time scales and at various levels of complexity. For example, instrumentation with advanced computer control and digital and spectral imaging approaches are revolutionizing microscopy methods, allowing spatially resolved spectroscopy and high throughput characterization of samples with enormous amounts of acquired data. Research and development from basic characterization of properties to "As someone who works diligently with resins for SLA, I can't help but believe that PAM will go beyond injection molding, tooling, and traditional manufacturing functionalities. I think that the current barrier lies in the development of AM-specific characterization for the materials we are using and would want to use in the future. This will enable new 3D printing processes and in turn, increase the possibilities for mass production."

\section{Maximilian Zieringer, Formlabs}

\section{- November 5, 2019, 3D Printing Industry} data analytics, prototyping, fabricating, and manufacturing are a must.

Metrology development and demonstration should be conducted with a close eye on materials and environmental, health and safety regulations, as well as sustainability, life cycle analysis, and disposal needs. The engagement of metrology equipment manufacturers with PAM stakeholders is important to ensure the needs of the community are heard as new input streams become widespread across the PAM supply chain and industries. Advanced measurement science will become increasingly import for detection of trace amounts of exposure to raw materials through occupational, environmental or accidental release, especially as volume streams of new and emerging materials grow. It will be essential to develop and deploy instruments and testing methods to measure the environmental fate of materials, develop safe material handling methods, and accurately measure environmental and health effects.

This panel focused on identifying the challenges and needs for advancing novel AM-specific characterization for the commercial production of uniform, high quality, stable and consistent PAM products in batch and high volumes. It is recognized that characterizations applicable to PAM products are necessary for input streams comprised of isolated materials and mixtures of materials that form composites. In addition, PAM input materials, and products, may be functionalized with moieties that offer electrical, thermal or mechanical capabilities. It is necessary to characterize the extent of these capabilities before, during (i.e., in situ), or after the manufacturing process.

\section{Current State and Technological Advances}

Adaptations to existing techniques, and entirely new tools, will be essential to meeting the needs of emerging materials for PAM and for characterizations during polymerization. Current available equipment in most cases is resolution-limited (e.g., temporal, spatial) and much greater measurement resolution and scale will be required for new applications to progress rapidly from laboratory research to commercial-scale manufacturing. Recent advances in AM-specific characterization are listed below: 
- Predictive transport kinetics model for photo-tunable hydrogel mechanical heterogeneity ${ }^{10}$

- Feedback-enhanced tomographic reconstruction for the high-resolution $(80 \mu \mathrm{m})$ volumetric production of centimeter-scale acrylic and silicone parts ${ }^{11}$

- Demonstration of rheological changes during photopolymerization at millisecond timescales and subvoxel length scales using atomic force microscopy technique, samplecoupled resonance photorheology 12

- Real-time interferometric monitoring and measuring of photopolymerization ${ }^{13}$

- Testing methodology ${ }^{14}$ to determine photopolymerization parameters including, $E_{c}$ (critical energy to initiate polymerization) and $D_{p}$ (penetration depth of curing light).

Poorly understood material mechanical and rheological properties during polymerization under relevant exposure/processing conditions and single-voxel length-scales limit advancements in part performance and throughput. A novel atomic force microscopy (AFM) technique, samplecoupled resonance photorheology (SCRPR) is capable of measuring rheological changes during photopolymerization at millisecond timescales and subvoxel length scales, thus enabling direct characterization of fast kinetic timescales. ${ }^{12}$ Success stories like these demonstrate how the metrology is a key enabling technology for the discovery, development and manufacturing of polymeric parts and systems and make it clear that real-time characterization tools, standardization and informatics are critical.

Materials testing standards. Forester ${ }^{15}$ recently summarized efforts by international standards organizations, namely the American Society for Testing Materials International (ASTM) and the International Standards Organization (ISO), on the development of standard test methods for mechanical properties and failure of polymers and polymer composites that are used in additive manufacturing processes. Briefly, these organizations have developed 55 standards for testing mechanical properties of polymers and composites. Most existing standards are applicable to testing AM produced parts, with some caveats. For example, some have limits in terms of their applicability, and others require guidance for modifications. These include geometric limits on test specimens, post-processing requirements to meet the specifications on parameters such as surface finish, material isotropy requirements and separating material properties from part performance,

\footnotetext{
${ }^{10}$ 2020: Callie I. Higgins, Jason P. Killgore, Frank W. DelRio, Stephanie J. Bryant, and Robert R. McLeod, Photo-tunable hydrogel mechanical heterogeneity informed by predictive transport kinetics model https://doi.org/10.1039/D0SM00052C Soft Matter, Advance Article 2020

11 2020: Loterie, D., Delrot, P. \& Moser, C. High-resolution tomographic volumetric additive manufacturing. Nat Commun 11,852 (2020). https://doi.org/10.1038/s41467-020-14630-4

12 2018: Callie I. Fiedler - Higgins Lewis M. Cox Frank W. DelRio Jason P. Killgore, Monitoring Fast, Voxel - Scale Cure Kinetics via Sample Coupled - Resonance Photorheology, https://doi.org/10.1002/smtd.201800275

${ }^{13}$ 2017: X Zhao and D W Rosen, Real-time interferometric monitoring and measuring of photopolymerization based stereolithographic additive manufacturing process: sensor model and algorithm, Meas. Sci. Technol. 28015001

${ }^{14}$ 2017: Joe Bennett, Measuring UV curing parameters of commercial photopolymers used in additive manufacturing, Additive Manufacturing, Volume 18, December 2017, Pages 203-212. https://doi.org/10.1016/i.addma.2017.10.009

15 2015: NISTIR 8059, Materials Testing Standards for Additive Manufacturing of Polymer Materials: State of the Art and Standards Applicability, Aaron M. Forester, http://dx.doi.org/10.6028/NIST.IR.8059
} 
and attention to specific application needs, such as temperature or immersion environment. Interestingly, a few of the composite standards are intended for materials containing high modulus fibers and as such are currently deemed "not applicable" to polymers in the current AM landscape. However, these could be applicable to PAM as reinforcements, such as fibers, become more prevalent in PAM applications as discussed in the previous section on emerging materials. An example list of properties requiring characterization methods and general approaches to make measurements are listed below.

\section{Properties and Characteristics Important for Measuring in PAM}

Mechanical: hardness, tensile strength, adhesion, elasticity, durability, rheology, force, strength, length, geometry and shape parameters (deformation), surface topography, final part dimensions, diffusion, dispersion, aging and longevity, viscosity, flow, fractures

Chemical: conversion, composition (atomic, elemental, molecular), charge, gelation, solubility, solvation, surface and interface chemistry and forces such as bonding, leachability, kinetics Electronic, photonic or magnetic: resistance, dielectric constant, refractive index, emissivity Process parameters: printability, build speed, light intensity, critical energy, light absorption depth, dose, dose rate, accumulated dose

\section{Measurement Approaches for PAM}

Microscopy: chemical atomic force microscopy, dynamic force microscopy, scanning transmission electron microscopy, scanning electron microscopy, aberration-corrected transmission electron microscopy, focused ion beam techniques, scanning near-field optical microscopy, scanning interferometric apertureless microscopy, photonic force microscopy Spectral: Electron energy-loss spectroscopy, X-ray scattering, real-time infrared spectroscopy Additional Common Tools: dynamic mechanical analysis, tensile/compression, rheometry, $\mathrm{X}$-ray computed tomography Looking Ahead Capabilities: Integrated approaches, in-situ measurements, data platforms, visualization, nanometer scales or less 


\section{Overarching Challenges}

Specific Overarching Challenges limiting novel AM specific characterization identified in the workshop with the associated needs to address each challenge are summarized below:

\begin{tabular}{|c|}
\hline Overarching Challenges and Needs in Novel AM Specific Characterization \\
\hline Lack of advanced characterization and standards \\
\hline $\begin{array}{l}\text { - Standard tests that rely on widely-available equipment and methods } \\
\text { - Test geometries that reveal inherent properties of heterogeneous, 3D parts (i.e. beyond } \\
\text { the dog bone) } \\
\text { - } \quad \text { Fundamental understanding of the printing processes and relationships of these to } \\
\text { - } \text { material properties } \\
\text { - } \quad \text { Modeling tools that can simulate 3D heterogenous geometries with multiple properties } \\
\text { - Predict geometric effects on properties } \\
\text { - Multi-scale models to correlate measured physical properties in situ with functional } \\
\text { - } \quad \text { Models to predict product functionality with aging (time, temperature, light exposure) } \\
\text { - Methods to probe layer-layer interface properties } \\
\text { - Non-destructive characterizations }\end{array}$ \\
\hline Rapidly evolving and dynamic hardware, software, and scalability needs \\
\hline $\begin{array}{l}\text { - Industry-wide collaboration to establish common standards with global stakeholder } \\
\text { community } \\
\text { - Models to identify resin/print property conditions during printing } \\
\text { - Quality control measures that can be implemented within the printing process }\end{array}$ \\
\hline Lack of clarity and specificity in environmental, health, and safety regulations \\
\hline $\begin{array}{l}\text { - } \quad \text { Characterization methods specific to EHS } \\
\text { - } \quad \text { Methods to support regulatory needs (validation of new materials) }\end{array}$ \\
\hline
\end{tabular}




\section{Recommended Research Directions}

The themes that emerged from the workshop were distilled into Recommended Research Directions with a series of solutions presented below. Bold items are included in the roadmapped solutions:

\begin{tabular}{|c|}
\hline Recommended Research Directions in Novel AM Specific Characterization \\
\hline Material Science and Engineering to Characterize PAM Materials \\
\hline $\begin{array}{l}\text { - Characterize dispersion and chemistry at the nanoscale to inform part performance } \\
\text { - Characterization of interfaces } \\
\text { - Incorporate additives to achieve functionality in situ } \\
\text { - Define universal energy unit standard for curing sources }\end{array}$ \\
\hline Advanced Computing for Materials Discovery and Process modelling \\
\hline $\begin{array}{l}\text { - Build a model to correlate printing conditions and final properties } \\
\text { - Integrate theory, modeling and experimental research - close coordination effort }\end{array}$ \\
\hline Measurement Science and Standards Development to Optimize PAM \\
\hline $\begin{array}{l}\text { - Enhance in situ process control and monitoring with feedback } \\
\text { - Develop AM-specific standards and calibrations } \\
\text { - Enable round robins, inter-laboratory comparisons } \\
\text { - Advance measurement science and tools to optimize quality control } \\
\text { - } \quad \text { Priorility testing over the full life cycle of the PAM process, including aging of products } \\
\text { - Non-destructive visualization of surfaces and sub-surfaces }\end{array}$ \\
\hline Environmental, Health, and Safety Regulations for Effective PAM Adoption \\
\hline $\begin{array}{l}\text { - Establish an industry-led framework to organize, build and maintain an inventory of } \\
\text { existing tools, tolerances, and applications for classes of PAM materials } \\
\text { - Determine critical measurands } \\
\text { - Identify progress, success, failures of science to support new instrumentation } \\
\text { - Capture gaps for problem areas with market pull } \\
\text { - Coordinate and link gaps to fundamental science R\&D }\end{array}$ \\
\hline Industry Collaborations to Enhance PAM Utility \\
\hline - Translate commercial needs into the fundamental science needed for PAM test artifacts \\
\hline
\end{tabular}




\section{Panel III: Industry Applications and Developing Markets}

\author{
Moderator: Tia Vialva, 3D Printing Industry \\ Rapporteurs: Mickey Fortune, RadTech \\ Panelists: Bob Gafvert, Carbon \\ Tom Bugnitz, Manufacturer's Edge \\ Denis Cormier, Rochester Institute of Technology \\ Robert McLeod, University of Colorado Boulder \\ Scott Turner, 3D Systems \\ Spencer Loveless, Dustless Technologies
}

\section{Panel Scope}

- Identify opportunities to advance market growth, including new niche markets, for more complex, functional PAM products

- $\quad$ Discuss big data needs for developing markets and increased competitiveness

- Consider ways to coordinate research efforts with industry needs

Many companies are developing inspiring new materials, process capabilities and equipment to produce functional applications and platforms for PAM. The community continuously seeks new ways to grow its markets and competitiveness through science and technology and small companies are recognized as the lifeblood of the industry.

One of the appealing aspects about PAM is that a functional part can be created over a short period of time. Depending on the size and complexity of the build, it can be as short as a few hours or a long as one business day. This short time allows businesses to make decisions about materials, product design, and manufacturability prior to full-scale production. Important mechanical, physical, and chemical properties as well as physical representations and esthetics of the product can be assessed on these rapid prototypes. For example, in an engineering application, effects of external loadings, torsion, tension, or pressure may be determined on model parts or tools. 16 All this information is especially useful for evaluating a new product for industry applications and developing markets, including niche markets.

\section{Parameters Influencing Speed of PAM Product Production}

- Physical and chemical properties of the resin

- Speed and resolution of the optical scanning system

- Power, wavelength and type of laser

- $\quad$ Spot size of the laser, recoating system, and post-curing process

- Presence of residual material (e.g. uncured resin, cured supports) that must be removed in post-process

It is recognized by the PAM community that big data is key to unlocking new PAM markets. Novel attempts are under development to advance mathematical models of the build process and algorithms to assess the progress, stability, and final characteristics of the constructed object. In addition, companies hoping to employ PAM for mass production will require greater process control, automation, and speed. There are many parameters influencing the speed of PAM product production (see table below). For example, today's technology does not have the speed necessary to compete with injection molding. Achieving high throughput capabilities will be essential for

\footnotetext{
${ }^{16}$ 2013: Thierry Chartier and Alexander Badev, Rapid Prototyping in Ceramics, In Handbook of Advanced Ceramics, Pages 489-524; http://dx.doi.org/10.1016/B978-0-12-385469-8.00028-9
} 
increasing PAM competitiveness. Large format systems and layerless systems are currently coming onto the market that are promising candidates to enable high-throughput PAM. ${ }^{17}$

\section{Current State and Technological Advances}

Prototyping is the dominant use of PAM and moving to the end use component production is the next big step for PAM. High throughput production capabilities are needed to take this big step. Current standout successes of PAM in a commercial context are found within the anatomy and medical fields. The benefits that have been delivered from 3D printed anatomical and medical structures include custom digital dental aligners, tissue scaffolds, and bone scaffolds. Furthermore, PAM of functional organs is emerging as a pathway towards organ manufacturing. Overall, PAM has the potential to positively impact public and individual health.

\begin{tabular}{|c|l|}
\hline \multicolumn{2}{|c|}{ PAM Application Segments } \\
\hline Medical & - Phantom models and surgical guides \\
& - Implants, prosthetics, orthotics \\
\hline - Rental & - Regenerative medicine \\
\hline - Implants (temporary or permanent) \\
\hline - Aligners \\
\hline - Surgical guides and production tools \\
\hline Automotive & - Small-batch Parts \\
& - Small components in weapons systems \\
\hline Consumer Products & - Hardware, tooling \\
& - Jectronics, semiconductors \\
\hline & - Eyewear \\
\hline & - Sootwear \\
\hline
\end{tabular}

Other fields seeing significant advances include products for physical fitness and sports. For example, the midsoles of running shoes can be tuned with custom cushioning specific to a runner's gait, enabling the safest and most effective energy return to the runner, which can lead to injury prevention. Related applications are emerging in sports protective equipment, where latticing can produce novel impact absorption properties in helmets and pads.

High-tech functional devices can also realize benefits from PAM. The fabrication of complex 3D microdevices, such as resonators and radio frequency (RF) filters used in high frequency operation circuits is an ideal application for PAM due the fabrication precision and spatial resolution of the technique. PAM offers a one-step printing capability for fully functional electronic devices, although this will require achieving nanoscale material functionalities in PAM processes as well as comprehensive sets of materials, design principles, and manufacturing guidelines. Smart materials,

\footnotetext{
17 2020: Polymer Additive Manufacturing (AM) Markets \& Applications, 2020-2029; Research and Markets Report Abstract on GlobeNewswire, March 9.
} 
such as chromic, self-healing, shape-memory, piezoresistive, and piezoelectric ones, are additional examples of potential smart material developing markets for PAM. ${ }^{18}$

Inkjet-specific applications hold promise but the ability to mix pigment materials is not trivial and requires much research and development for each application. There are volume and reliability limits that must be overcome. Because formulations for inkjet are so application specific, it is hard to consider investments in this area unless it is based on a specific customer need and funded through that customer. This approach is still not applicable beyond custom applications that are low volume. However, there is some viability of PAM to take on high-value inkjet molding applications. For example, conductive printing is an exciting tool for printing circuits, though this is still in the research phase for the PAM industry.

Redesigning complex, multi-part assemblies produced by injection molding and secondary operations into one 3D printed part is a key future direction to consider for PAM. Removing the costs with individual molding will free resources for new R\&D in 3D printing applications. Specific solutions towards this approach will be accelerated through computational platforms. Having these platforms will enable realms of data to be available to fuel the industry, provided such platforms are shared use. As these platforms grow and develop, cybersecurity is increasingly important. Data needs to be protected to keep intellectual property safe. Cybersecurity frameworks and standards are important now and going forward for the PAM industry.

\footnotetext{
${ }^{18}$ 2019: State-of-the-Art and Future Challenges of UV Curable Polymer-Based Smart Materials for Printing Technologies, Cristian MendesFelipe, Juliana Oliveira, Ikerne Etxebarria, José Luis Vilas-Vilela, and Senentxu Lanceros-Mendez, Adv. Mater. Technol. 2019, 4, 1800618; https://doi.org/10.1002/admt.201800618
} 


\section{Overarching Challenges}

Specific Overarching Challenges limiting sustainable, hybrid, and emerging materials for PAM identified in the workshop with the associated needs to address each challenge are summarized below:

\begin{tabular}{|c|}
\hline Overarching Challenges and Needs in Industry Applications and Developing Markets \\
\hline Limits in materials and chemistry \\
\hline $\begin{array}{l}\text { - New, application-specific materials with predictable performance } \\
\text { - Materials for larger volume builds }\end{array}$ \\
\hline Lack of advanced characterization and standards \\
\hline $\begin{array}{l}\text { - Monitoring dynamics of build and assembly } \\
\text { - } \quad 3 D \text { visualization of surface and interior composition and geometry } \\
\text { - } \text { Access to nanoscale measurements } \\
\text { - Precision control of all steps in printing process } \\
\text { - Rheology testing, training } \\
\text { - }\end{array}$ \\
\hline Rapidly evolving and dynamic hardware, software, and scalability needs \\
\hline $\begin{array}{l}\text { - } \\
\text { - } \quad \text { Better understand and control build speed } \\
\text { - } \quad \text { Simplify multi-part assemblies into single-part designs } \\
\text { - } \quad \text { Bringing in younger generation to start manufacturing companies } \\
\text { - } \quad \text { PAM-specific non-destructive evaluation methods } \\
\text { - } \quad \text { Manufacturing-scale maker-spaces for PAM } \\
\text { - } \quad \text { Translate commercial needs into fundamental science questions that need answers via } \\
\text { - } \quad \text { modeling and theory } \\
\text { - } \quad \text { Predict performance of novel PAM resin formulations, composites and additives } \\
\text { - } \quad \text { Model quality control for homogeneity and purity calculations } \\
\text { - } \quad \text { Correlating measured properties of input materials to performance in final products } \\
\text { - } \quad \text { Awareness of PAM in existing products, capabilities }\end{array}$ \\
\hline Lack of clarity and specificity in environmental, health, and safety regulations \\
\hline - Manage environmental impact \\
\hline
\end{tabular}




\section{Recommended Research Directions}

The themes that emerged from the workshop were distilled into Recommended Research Directions with a series of solutions presented below. Bold items are included in the roadmapped solutions:

\begin{tabular}{|c|}
\hline Recommended Research Directions in Industry Applications and Developing Markets \\
\hline Material Science and Engineering to Characterize PAM Materials \\
\hline $\begin{array}{l}\text { - Discovery of next generation polymeric blends to fuel market growth in PAM } \\
\text { - Investigate smart and multi-functional material opportunities }\end{array}$ \\
\hline Advanced Computing for Materials Discovery and Process modelling \\
\hline $\begin{array}{l}\text { - Address scale-up challenges for both process control and high-throughput manufacturing } \\
\text { and measurement capabilities } \\
\text { - Develop modeling tool kits to simulate new classes of materials and properties } \\
\text { - Develop approaches to extract critical and strategic information from large data sets } \\
\text { - Address regulatory needs through shared databases of tools for pre-competitive use }\end{array}$ \\
\hline Measurement Science and Standards Development to Optimize PAM \\
\hline $\begin{array}{l}\text { - Evaluate hardware and software challenges for design, control and integrated } \\
\text { measurement } \\
\text { - Integrate non-contact inspection capabilities with needed tolerance } \\
\text { - Create advanced multi-scale computing capabilities for new equipment and materials } \\
\text { - } \quad \text { Inteate modeling tools to enable robust manufacturing capabilities } \\
\text { - Integri-scale models and tools to enable control of manufacturing processes }\end{array}$ \\
\hline Environmental, Health, and Safety Regulations for Effective PAM Adoption \\
\hline $\begin{array}{l}\text { - Support data systems for PAM registries and regulations } \\
\text { - Address regulatory needs through shared databases of tools for pre-competitive use }\end{array}$ \\
\hline Industry Collaborations to Enhance PAM Utility \\
\hline $\begin{array}{l}\text { - } \text { Translate commercial needs into R\&D efforts } \\
\text { - Advance manufacturing capabilities in PAM } \\
\text { - Support collaborations between small- and large-scale PAM industry adopters } \\
\text { - Engage university engineering programs with industry R\&D for large and small companies } \\
\text { - Entrepreneurship and technology transfer training into academic programs specific to } \\
\text { starting small PAM manufacturing companies }\end{array}$ \\
\hline
\end{tabular}




\section{Moderator: Tom McKeag, The Berkeley Center for Green Chemistry}

Panel IV: Health, Safety, and Regulation in AM

Rapporteurs: Dianne Poster, NIST

Panelists: Treye Thomas, Consumer Product Safety Commission

Gary Roth, National Institute for Occupational Safety and Health

Alexandria Stanton, U.S. Environmental Protection Agency

Michael Brady, 3D Systems

Stephanie Benight, Tactile Materials Solutions, Inc.

\section{Panel Scope}

- Assess regulations needed to protect workers and consumers

- Discuss safety issues for endproducts

- Consider gaps in home-use

- Consider fundamental science and measurement capabilities required to obtain health, safety and environmental information

- Applications and maker-space needs

- Outline distinct roles of different regulatory agencies to PAM

As a novel, advanced technology making use of new materials, including nanomaterials, stakeholders agree PAM materials and products pose concerns regarding potential consumer, worker, and environmental health effects. There is also a paucity of regulations for all aspects of the supply chain. These concerns and gaps must be addressed to enable the widespread economic and societal benefits that PAM offers to businesses and consumers. Fundamental science, accurate and efficient risk assessment and management, cost-benefit models and enhanced public education and confidence are required.

More specifically, a better understanding of the life-cycle implications of PAM materials and products for the environment and human health and safety is needed. Measurements, testing and data will be critical to this endeavor for the industry. For example, predicting and detecting trace levels of exposure to PAM materials resulting from medical, occupational, environmental, or accidental release will be necessary through advanced modeling and measurement capabilities to support guidance and regulations. Instrumentation and methods will be needed to accurately follow the biological transformations and environmental fate of PAM materials and products, develop safe materials handling methods for both consumers-as-manufacturers and industrial scenarios, and to accurately measure the near- and long-term effects.

Given the size of the PAM industry and its anticipated growth into new markets, protecting human health, safety, and the environment must be considered. EHS considerations must be an integral component as PAM materials and products are researched, developed, manufactured, used, and disposed. There are currently a number of uncertainties, such as how volatile vapors might be transported in air during research or manufacturing; how surfaces of finished products might react with the environment or in contact with consumers; how insoluble particles in material streams are distributed and deposited; and whether final parts degrade and accumulate. Questions such as these will require fundamental focused attention by the PAM community as the technology continues to advance and enable high volume production either industrially or via consumer manufacturing.

As science and engineering advance PAM equipment, processes, and product design, fundamental R\&D may open doors to reduce or eliminate substantiated health and environmental risks. The benefits from the field of PAM and public wide-spread adoption of PAM products will depend on a reliable scientific capability to understand and proactively manage potential hazards to 
human health and the environment (indoor and outdoor). Indoor environments need to consider both industrial and residential settings. Developing this capacity will require a coordinated effort of many disciplines from a variety of organizations across government agencies and through its collaborative frameworks with industry and academia. Public and environmental health advocate organizations will be equally essential in this coordinated effort to enhance public acceptance and use of PAM in a responsible manner. A coordinated framework will be integral to progressing risk evaluation and risk mitigation for the

PAM industry.

With that said, PAM is novel, young and rapidly growing. The increased pace and complexity of technological developments are posing challenges to the existing regulatory system. In today's U.S. market for AM, the existing regulatory system is overseen by the Environmental Protection Agency (EPA), the Food and Drug Administration (FDA), the Occupational Safety and Health Administration (OSHA), the National

"As with any evolving technology, 3D printing raises a host of environmental legal questions, surrounding, for example, hazardous waste, air emissions, recycling and chemical disclosure, just to name a few. Recently, 3D printing has caught the attention of the US Environmental Protection Agency (EPA)."

-- Lauren A. Bachtel \& Alexander Woo, The Nickle Report, December 10, 2019 Institute of Occupational Safety and Health (NIOSH), and the Consumer Product Safety Commission (CPSC). The significant lack of data on the health effects due to exposure to PAM formulations and products hinders the identification of potential concerns, which inherently, and unfortunately, may dampen innovation. This scenario mirrors the early stages of the nanotechnology field about fifteen years ago. Experts then argued that health and safety research and regulation must be conducted concurrently with product development to keep pace with the speed of innovation. However, on the flipside, opponents argued such research requires time to understand, and more importantly, observe, potential detrimental effects, such as hospitalizations, disease, or death, and as a result effects would outpace the commerce activity, i.e., the damage will already have been done by the time research catches up to the trade. ${ }^{19}$ The same arguments apply to today's developing field of PAM.

It is clear safety and health guidelines are needed to ensure the responsible development of PAM products and the safety of workers, consumers and the environment. Some experts suggest that safety concerns about formulations and products can be managed under existing laws and regulations, while others see that approaches founded upon fundamental research, science and data may be necessary as the technology advances. In general, existing regulatory agencies provide appropriate mechanisms to address the current safety needs for PAM (human and environmental) through their existing authorities, although as new PAM formulations advance and worker spaces evolve from an industrial setting to an at-home environment, new mechanisms may be required. In these cases, new mechanisms should be developed in a consistent manner across regulatory agencies in collaboration with standards developing organizations. For example, as metrics become more developed and integrated into the PAM supply chain through research and characterization data, new voluntary consensus standards may be developed based on these data, including

\footnotetext{
${ }^{19}$ 2011: John F. Sargent Jr., “Nanotechnology and Environmental, Health, and Safety: Issues for Consideration”, Congressional Research Service, RL 34614 (Report)
} 
standard test methods. These standards may be incorporated by reference into new regulation formally or alternatively may be adopted industry-wide as best practices through a certification process, i.e., a publicly recognized mark of quality that ensures consumer confidence. The "Energy Star" program is an example of such an approach, which is a voluntary program managed by the EPA designed to help manufacturers and consumers make choices about products to enable cost savings and climate protection through energy efficiency.

Additive manufacturing hazards vary widely depending on the technology and materials used. Examples of these hazards include: ${ }^{20}$

- Solvents and resins may emit volatile organic compounds, and some processes may promote emission of volatile organic compounds

- Dermal exposure to hazardous materials is possible

- Some flammable or combustible liquids and powders may create a risk of fire or explosion

- Machine support and maintenance, loading of build materials, and handling of products may create risks of mechanical or ergonomic injury and stress

- Exposures to organic polymers, particulate matter, corrosive cleaning agents and noise may also be possible. ${ }^{21}$

The above issues reflect the needs of PAM and can only be addressed through federal investments in basic research, federal regulation, and international engagement. In the field of nanotechnology, coordination of research, regulations, standards and enforcement is through collaborative global models to protect workers and consumers as products are produced along global supply chains and sold in industrial and commercial markets worldwide. Industry, government, academic, and public bodies participate to promote the responsible development of new materials and products and share information to address environmental, health, safety, societal and ethical issues as nanotechnology evolves. ${ }^{22}$ As PAM increasingly incorporates new materials into its product streams, including nanomaterials, such an approach can clearly support the development of tools and methods to identify, prioritize, and manage strategies for specific research to enable risk analysis and regulatory decision-making for PAM materials and products. Adequate and timely safety guidelines and regulations must be developed and put into practice to ensure that PAM R\&D is sustainable, cost effective, and of long-term benefit to consumers and endusers.

In conclusion, data are essential for any health, safety and regulation efforts, especially those supporting biological effects, sustainability and life-cycle planning. Moreover, the need for coordinated research, funding, and management by government agencies and stakeholders is continuous. This continuity function has been essential and successful for the nanotechnology industry. ${ }^{23}$ Similarly in the PAM industry, refinements and advances in methods to detect, measure, and assess PAM materials and products in realistic workplace, consumer and environmental

\footnotetext{
${ }^{20}$ 2020: NIOSH, “Additive Manufacturing/3D Printing” (Webpage)

${ }^{21}$ 2016: Tim Ryan and Daniel Hubbard, "3-D Printing Hazards: Literature Review \& Preliminary Hazard Assessment" American Society of Safety Engineers, Professional Safety, 61:06 (Article)

22 2011: John F. Sargent Jr., "Nanotechnology and Environmental, Health, and Safety: Issues for Consideration", Congressional Research Service, $\underline{\text { RL } 34614}$ (Report)

${ }^{23}$ 2017: Highlights of Recent Research on the Environmental, Health, and Safety Implications of Engineered Nanomaterials, National Nanotechnology Initiative (Document)
} 
conditions will continue to be necessary to develop and maintain effective management strategies. In addition, government agencies must participate in these efforts under the auspices of their mission profiles to ensure success.

As mentioned above, agencies with regulatory authorities applicable to PAM are EPA, FDA, OSHA, NIOSH, and CPSC. Agencies supporting R\&D programs and grant funding programs are equally essential to PAM health, safety and regulation needs. These include EPA, NIST, the National Science Foundation (NSF), the Department of Energy (DOE), the Department of Defense (DOD), and the Department of Health and Human Services (including OSHA, NIOSH and CDC). Representatives from EPA, NIOSH, CPSC, NIST and DOD were present at the workshop.

\section{Current State and Technological Advances}

In the PAM field, existing scientific health, safety and regulation frameworks are available through materials, chemical, biological and occupational programs in the agencies identified above. Each agency's mission and authorities are elaborated on further below in this section. In general, PAM companies are proactively seeking to promote the technology and the responsible development of future applications through R\&D and engagement with science and regulatory agencies and academia. Networking is enhanced through trade organization events and meetings such as those provided by RadTech. For example, RadTech has developed and disseminated information for the proper handling of 3D printing materials and plans to build on this effort as a result of the collaboration developments from the PAM 2019 workshop, in partnership with PAM stakeholders. RadTech has pledged to commit resources to help lead efforts to develop the necessary data and information required by regulators and others that may have health and safety concerns about PAM as follow up activities to the PAM 2019 workshop.

Fast-paced changes in PAM input streams with new formulations, rapid advances in new consumer products, and increased home-based manufacturing make staying connected and up-todate a challenging, and often daunting, process. Having a centralized, coordinated neutral body to help expedite this process would be beneficial and offer cost-savings. All parties could conceivably participate and contribute more readily to methods, standards, guidance and regulatory developments while simultaneously maximizing information sharing and use, i.e., best practices or lessons learned, in a pre-competitive environment.

In the area of disposal, EPA, NIOSH and CPSC generally cover about $90 \%$ of the regulations regarding the disposal of resins or printed parts. EPA covers oversight for the raw materials with respect to regulation (not raw materials in research, but chemicals used for producing manufactured products). NIOSH oversees workplace safety, including testing. In addition, NIOSH regulates respirators and some mining equipment, but OSHA regulates health and safety of workers. NIOSH does research to support OSHA. There are multiple levels of regulations involved with respect to what workers are permitted to be exposed to and with respect to the equipment that is being used. CPSC covers the products for input streams (not the individual chemicals, but the products that are in commerce that contain the chemicals), the device and the finished product for homes and schools. A classic example is the presence of lead in consumer toys. In PAM, the chemical nature of the raw materials and residues (e.g. residual monomers and photoinitiators) results in regulations for residual monomers and photoinitators, consumer health (CPSC) and environmental protection (EPA). CPSC does not have pre-market approval for any product. Pre-market approvals are very specific for the type of product entering commerce, for example any medical device must be premarket approved through FDA. In the next section, more information is provided on the regulatory roles of EPA, FDA, OSHA, NIOSH and CPSC. 
International regulations, such as REACH (Registration, Evaluation, Authorization and Restriction of Chemical) ${ }^{24}$ of the European Parliament, are important. REACH has the slogan "no data no market" demonstrating the responsibility to manage the risks from chemicals and to provide safety information on the substances falls on industry, similarly to EPA's chemical mandates. In REACH and EPA regulations, manufacturers and importers are required to gather information on the properties of their chemical substances, which will allow their safe handling.

\section{Current regulatory agencies roles in health, safety and regulation in AM}

Environmental Protection Agency (EPA). The EPA has both a research function and a regulatory function. EPA research plays an important part in EPA's mission to protect human health and the environment by evaluating new and emerging materials as they enter the market. EPA science supporting AM is emerging. For example, EPA scientists (with CPSC) are investigating VOC emissions from 3D printers as a function of additives within the 3D printing filament. This work is the beginning of a focused EPA effort to support the science and regulatory needs for 3D printing. ${ }^{25}$ Additional activities will be through collaborative EPA studies with other agencies, such as CPSC and $\mathrm{NIOSH}$ and through EPA's extensive extramural grant programs.

EPA regulations are authorized by Congress to explain the technical, operational, and legal details necessary to implement laws. Regulations are mandatory requirements that can apply to individuals, businesses, state or local governments, non-profit institutions, or others. Manufacturers are required to provide data to EPA through various channels to determine risks, and EPA ensures this through regulation and enforcement. Regulatory authorities pertinent to PAM are the Clean Air Act (CAA, 42 U.S.C. 7401 et seq), Clean Water Act (CWA, codified generally as 33 U.S.C. et seq.), Federal Insecticide, Fungicide, and Rodenticide Act (FIFRA, 7 U.S.C. §136 et seq.) and Toxic Substances Control Act (15 U.S.C. 2601 et seq.). There may be additional environmental statutes, executive orders, or policy and guidance documents applicable to PAM that provide oversight to pre-manufacturing, new, significant new use, or existing chemical actives.

Food and Drug Administration (FDA). The FDA is an agency within the U.S. Department of Health and Human Services. The core functions of the agency are medical products and tobacco, foods and veterinary medicine, global regulatory operations and policy, and operations. FDA has developed guidance ${ }^{26}$ to provide technical considerations specific to devices using additive manufacturing. The FDA recognizes that the rapid technological advancements and increased availability of AM fabrication equipment are encouraging increased investment in the technology and its increased use by the medical device industry.

The FDA guidance is intended to outline technical considerations associated with AM processes, and recommendations for testing and characterization for devices that include at least one additively manufactured component or additively fabricated step. Regulated products pertinent to PAM are medical devices, tissues or orthopedics. As soft polymeric nanomaterials, such as gels, become more prevalent in PAM, these will likely be regulated under the product category of

\footnotetext{
${ }^{24}$ 2019: European Commission, "REACH" (Webpage)

25 2020: David Sparkman, “EPA Looks at 3D Printing Emissions”, EHS Today, January 16 (Article)

${ }^{26}$ 2017: FDA, Center for Devices and Radiological Health, “Technical Considerations for Additive Manufactured Medical Devices", Docket number FDA-2016-D-1210 (Webpage and document)
} 
biologics. FDA oversight for AM resources in response to the COVID-19 pandemic has been instrumental for manufacturers. The FDA is a Gold Member of America Makes, the National Additive Manufacturing Innovation Institute. America Makes and the FDA are now partnering "to help ensure the additive manufacturing industry can effectively and safely meet the needs of America's health care workers on the front lines of the Coronavirus crisis." ${ }^{27}$

Occupational, Safety and Health Administration (OSHA). With the Occupational Safety and Health Act of 1970, Congress created OSHA to ensure safe and healthful working conditions for working men and women by setting and enforcing standards and by providing training, outreach, education and assistance. OSHA is part of the U.S. Department of Labor. The OSHA Act covers most private sector employers and their workers, in addition to some public sector employers and workers in the 50 states and certain territories and jurisdictions under federal authority. While OSHA creates and enforces regulations, NIOSH focuses its attention on pushing the scientific field of occupational safety forward. They share the results of their research through robust education programs. They even provide workplace training to improve safety at businesses.

National Institute of Occupational Safety and Health (NIOSH). NIOSH is a research and education institution, not a legal enforcement agency. NIOSH recognizes the promise of AM technologies and is performing research to understand and minimize its potential health and safety risk to the American worker and is supporting the safe and responsible deployment of the technology. NIOSH is conducting research on safe additive manufacturing to identify knowledge gaps, to develop robust science necessary to advance understanding in the field, and to best apply these developments in order to protect the American worker. $28,29,30$

Consumer Product Safety Commission (CPSC). CPSC is an independent federal regulatory agency formed in 1972 with a mission to protect the public against unreasonable risks of injury or death from consumer products through education, safety standards activities, regulation, and enforcement. CPSC works to save lives and keep families safe by reducing the unreasonable risk of injuries and deaths associated with consumer products and fulfilling its vision to be the recognized global leader in consumer product safety. CPSC does this by:

- Developing voluntary standards with standards organizations, manufacturers and businesses;

- Issuing and enforcing mandatory standards or banning consumer products if no feasible standard would adequately protect the public;

- Obtaining the recall of products and arranging for a repair, replacement or refund for recalled products;

- Researching potential product hazards;

- Informing and educating consumers directly and through traditional, online, and social media and by working with foreign, state and local governments and private organizations; and

\footnotetext{
${ }^{27}$ 2020: Sarah Goehrke, "FDA Oversight Emerges For Additive Manufacturing Resources In Pandemic Response", Forbes, March 14 (Article)

28 2020: NIOSH, “Additive Manufacturing/3D Printing” (Webpage)

29 2019: Kevin Druley, “3D printing and worker safety, Exploring the hazards of this emerging technology", Safety + Health, April 28 (Article)

${ }^{30}$ 2020: NIOSH, “3D Printing with Filaments: Health and Safety Questions to Ask”, DHHS (NIOSH) Publication Number 2020-115 (Webpage)
} 
- Educating manufacturers worldwide about our regulations, supply chain integrity and development of safe products.

CPSC does not endorse or recommend specific brands of products. CPSC relies on its main statutes, the Consumer Product Safety Act (CPSA) and the Federal Hazardous Substances Act (FHSA) for most of its jurisdiction.

CPSC recently released a report on the safety concerns associated with 3D printed consumer products. Briefly, two broad hazard areas have been identified: (1) hazards posed by use of PAM process, and (2) hazards posed by the printed products. Each of these hazard areas includes potential fire and combustion hazards, electrical hazards, chemical hazards, and mechanical hazards. In addition, staff has concerns that PAM consumer products-particularly children's products-may not meet applicable regulations or voluntary standards, due to the PAM manufacturer's potential unfamiliarity with federal regulations and voluntary standards applicable to those products, especially for small and micro-producers that are new entrants to the market. Staff also has concerns about the potential health effects of materials used for PAM and the potential for consumer exposures. To this end, staff is working along multiple fronts, including voluntary standards development and research. Staff has developed interagency agreements with federal partners to investigate the potential health effects of materials used in, and chemical and particulate (including nanoparticle) emissions from, PAM and potential consumer exposure. ${ }^{31}$

${ }^{31}$ 2020: CPSC, "Safety Concerns Associated with 3D Printing and 3D Printed Consumer Products", May 6 (Report) 


\section{Overarching Challenges}

Specific Overarching Challenges limiting sustainable, hybrid, and emerging materials for PAM identified in the workshop with the associated needs to address each challenge are summarized below:

\section{Overarching Challenges and Needs in Health, Safety, and Regulation in AM}

\section{Limits in materials and chemistry}

- Formulate resins with reduced health risks for manufacturers and end-customers

- Formulate resins with reduced environmental impact

- Formulate resins that facilitate improved safety and reliability of final parts

Lack of clarity and specificity in environmental, health, and safety regulations

- Guidelines for the safe handling of materials, equipment and products for industry and public

- Guidance for home-based printing and manufacturing

- Mechanism for communicating risk via consistent labeling requirements for chemicals and products

- Better information on sustainability

- Reliable methods to detect vapors or particles specific to PAM in the workplace

- Databases on health effects of classes of materials

- Public guidance for proper disposal, reuse and recycling of PAM resins and parts

- Frameworks for consumer life cycle assessments

- Identification of research and models for toxicological assessments

- Integration of available research on acrylates with long term assessments through models and R\&D

- Incorporation of environmental studies in long-term health effect studies - ecosystem effects, fate and transport 


\section{Recommended Research Directions}

The themes that emerged from the workshop were distilled into Recommended Research Directions with a series of solutions presented below. Bold items are included in the roadmapped solutions:

\begin{tabular}{|c|}
\hline Recommended Research Directions in Health, Safety, and Regulation in AM \\
\hline Material Science and Engineering to Characterize PAM Materials \\
\hline $\begin{array}{l}\text { - Interface with systems biology approaches } \\
\text { - Grow capabilities in biomaterials R\&D } \\
\text { - Create centralized databases on biocompatibility and biodegradation of PAM-specific } \\
\text { polymers and additives }\end{array}$ \\
\hline Advanced Computing for Materials Discovery and Process modelling \\
\hline $\begin{array}{l}\text { - Develop empirically-informed theory, modeling, and simulations of PAM } \\
\text { - Identify and encourage results to be published for poorly characterized/tested materials } \\
\text { - Establish a data base of testing results for PAM-specific materials that is a searchable tool }\end{array}$ \\
\hline Measurement Science and Standards Development to Optimize PAM \\
\hline $\begin{array}{l}\text { - } \quad \text { Develop acrylate chemical substitutions } \\
\text { - } \quad \text { Develop artifact standards and standard tests and testing methods specific to EHS } \\
\text { - } \quad \text { Engage international community for cross-border regulation needs } \\
\text { Define calibration and standards needs }\end{array}$ \\
\hline Environmental, Health, and Safety Regulations for Effective PAM Adoption \\
\hline $\begin{array}{l}\text { - Define minimal dataset for risk assessment, exposure } \\
\text { - Develop framework for product stewardship } \\
\text { - Develop simulation tools to describe quantitative connections between PAM materials' } \\
\text { properties and biological effects }\end{array}$ \\
\hline Industry Collaborations to Enhance PAM Utility \\
\hline $\begin{array}{l}\text { - Establish multi-stakeholder framework for coordinating safe handling of materials, } \\
\text { equipment and products with collaboration across stakeholders } \\
\text { Develop mechanism to facilitate and encourage academic research and strengthen } \\
\text { collaboration with industry }\end{array}$ \\
\hline
\end{tabular}




\title{
Panel V: Moon-shot Ideas in AM
}

\author{
Moderator: Simon Lancaster, Apple \\ Rapporteurs: Callie Higgins, NIST \\ Panelists: Charlie Wood, Fast Radius \\ Will Tashman, Uncountable \\ John La Scala, Army Research Laboratory \\ Maxim Shusteff, Lawrence Livermore National Lab \\ Blake Perez, nTopology \\ Jed Brown, University of Colorado Boulder
}

Panel Scope

- Define moonshot in context of PAM

- Consider new processes, materials and products for moonshots

- Determine challenges facing PAM today that moonshot could solve

- Highlight current moonshot ideas and efforts in PAM

Moonshots are an aspirational goal that offer major "jumps" rather than incremental improvements. For example, achieving revolutionary science and technology products that were once imagined only in the movies is what makes a true moonshot. However, while a moonshot's success is the actual achievement itself, such as the 1969 landing of the Apollo 11 mission on the moon, the externalities that take place along the way are equally moonshot worthy. Overall, a moonshot is something that sustainably revolutionizes an industry.

Additive manufacturing in its current state is not considered a moonshot per se because it is still developing, but achievements are happening so intensely, they are revolutionizing the industry and offering capabilities to the everyday lives of the public in a sustainable fashion, i.e., with every advance there is an equal advance in new opportunities, capabilities or products. Already the industry has moved from thin layer forms to much bigger structures that are not confined to substrates. It is anticipated PAM will generate a "large jump" in technology, and this time scale will not be decades from today but rather in the next few years. The industry is moving and growing quickly.

The early adoption phase of PAM has moved to substantially impacting the public. Even those who are not familiar with additive manufacturing, materials, or technology are producing products for their own entertainment, hobbies, or as one-person manufacturers with available printing materials and equipment. Moreover, industry is actively looking to additive manufacturing for solutions within existing markets and access to new markets. The computer giant Hewlett Packard recently stated "...the technology will help fuel the arrival of automated assembly, with industries seamlessly integrating multi-part assemblies including combinations of both 3D printed metal and plastic parts". ${ }^{32}$ Large jumps in progress are happening every few months in the field, which will eventually cumulate to the anticipated "big" moonshot jump. An industry-wide jump is moonshot worthy too. For example, PAM becoming a multi-industry manufacturing technique that everyone learns and uses similarly to injection molding is surely a moonshot. Moving from injection molding or machining of multiple parts to a "one-single part" production via PAM would be revolutionary. An advantage of PAM is in its potential flexibility and ability to offer adaptability to different materials or requirements. Equipment manufacturers will play a key role in engineering flexible and adaptable capabilities in the industry.

Modeling and simulations will be key to achieving moonshots in PAM. Computer-assisted capabilities to predict and visualize the design of new materials, products, and the printing process,

32 2020: Sparkman, D., “EPA Looks at 3D Printing Emissions”, EHS Today, January 16 (Webpage) 
as well as the modeling of health effects, risk assessment and toxicology, are limited at best in the current state of PAM and need to advance. Similarly, deep machine learning is important to propel advances in technology towards achieving PAM moonshots. Machine learning in the printing process could break the barriers to realizing seamless printing and the high-volume production of defectfree products. Defects are often the result of weak regions in a printed part and lead to poor performance or failure. Enabling sophisticated artificial intelligence to prevent defects could lead to high volume production of PAM parts in markets where tolerance levels are extremely high, such as in aerospace, and could lead to PAM products literally contributing to a moonshot.

Lastly, the concept of moonshot "death" is intriguing. Death of a moonshot is generally not cost related as decisions have already been made to "take a shot" to achieve success and investments have been poured into the concept R\&D. However, somewhere along the way, the mission dies. Moonshots die prematurely for several reasons. In some cases, it is just plain human nature i.e., it just gets too hard, staff burn out and the ideas die. In other cases, companies get distracted by the success of externalities which seem more feasible to follow at the time due to the "mini-successes" achieved along the way to the target. Companies eventually lose sight of the moonshot and forget about it. In other cases, market interests change and the public may demand something entirely different than what was originally designed as the target. For example, when the input stream for a moonshot application contains a chemical that is originally thought to be nontoxic but is found to be hazardous, the mission goal will die. In some cases runaway costs may be a factor for R\&D teams to cut their losses and move on.

\section{Current State and Technological Advances}

Progress toward moonshots in PAM is being made as structures become more intricate and larger and are no longer being confined to a substrate. Moonshots are not being declared due to the lack of known intentions within the progress areas. Impressive technologies are advancing, especially in the medical field, but the declarations have not been made. Moonshots are difficult to identify and even more difficult to predict.

One example of an anticipated moonshot in PAM is the 3D printing of batteries. It is difficult to create materials that are suitable for the process, either by extrusion of a solid or a liquid or by the polymerization of liquid. Batteries contain electroactive materials that are reactive. The complexity of the electrochemistry and the physical nature of anodes and cathodes requires extensive chemical and material measurements. For example, crystallography and porosity become important. Requirements of a printed product include sustainable electrical conductivity, controlled chemistry, chargeability and safety. In PAM today, there are lattices or meta-materials broadly being used with architecture designs to overcome limits of materials or bring new capabilities on structuring the components in batteries, anodes, cathodes, and separators, whether it's membrane or support for the catalyst. The ability to design batteries for 3D printing that can be recycled may be the driving factor to achieving this moonshot. ${ }^{33}$

Success in functioning organ 3D printing will alleviate the crisis of organ shortages around the world. Models and simulations will be key to finding where the problems are in the current technology and will help break the printing and design barriers to moonshot achievements. It is not unfathomable to realize battery or functional organ printing in the future for PAM.

\footnotetext{
${ }^{33}$ 2019: “IS 3D printing the future of battery design?”, MIT Technology Review Post, December 22 (Webpage)
} 


\section{Overarching Challenges}

Specific Overarching Challenges limiting sustainable, hybrid, and emerging materials for PAM identified in the workshop with the associated needs to address each challenge are summarized below:

\begin{tabular}{|c|}
\hline Overarching Challenges and Needs in Moonshot Ideas \\
\hline Limits in materials and chemistry \\
\hline $\begin{array}{l}\text { - Materials for printing high-performance, functional devices (e.g. batteries, } \\
\text { semiconductors, organs) } \\
\text { - Material discovery via high performance computing }\end{array}$ \\
\hline Lack of advanced characterization and standards \\
\hline $\begin{array}{l}\text { - } \quad \text { Adaptable machine learning } \\
\text { - } \quad \text { Prediction of materials' properties (strength, toughness) } \\
\text { - } \quad \text { Moving towards one-step printing by removing post processing requirements } \\
\text { - } \quad \text { Fundamental R\&D to understand and prevent defects } \\
\text { - Understanding post-printing effects due to light, gravity and temperature }\end{array}$ \\
\hline Rapidly evolving and dynamic hardware, software, and scalability needs \\
\hline $\begin{array}{l}\text { - Approaches to simulate fast design cycle } \\
\text { - Solutions to improve PAM throughput by orders of magnitude } \\
\text { - Increased speed and precision in printing } \\
\text { - Changing perception that PAM is a real manufacturing process }\end{array}$ \\
\hline
\end{tabular}




\section{Recommended Research Directions}

The themes that emerged from the workshop were distilled into Recommended Research Directions with a series of solutions presented below. Bold items are included in the roadmapped solutions:

\begin{tabular}{|c|}
\hline Recommended Research Directions in Moonshot Ideas \\
\hline Material Science and Engineering to Characterize PAM Materials \\
\hline $\begin{array}{l}\text { - Develop capabilities for new design processes and approaches to work with new } \\
\text { materials }\end{array}$ \\
\hline Advanced Computing for Materials Discovery and Process modelling \\
\hline $\begin{array}{l}\text { - Launch major efforts in computing and informatics to predict print process } \\
\text { - Create programs to generate fundamental data needed for models and integrate } \\
\text { modeling and experimental programs with collaboration among stakeholders } \\
\text { - Develop programs in machine learning and adaptability }\end{array}$ \\
\hline Measurement Science and Standards Development to Optimize PAM \\
\hline $\begin{array}{l}\text { - Expedite materials characterization via machine learning } \\
\text { - Integrage dimensional metrology and calibration for precision manufacturing } \\
\text { - Capabilities to maintain, measure, or move to a position or follow a path with a level of } \\
\text { accuracy with input beams } \\
\text { - Grow expertise and capabilities in optomechanical engineering with factory floor pilots } \\
\text { - Develop calibrations and standards, including test methods and artifacts, for precision } \\
\text { manufacturing }\end{array}$ \\
\hline Environmental, Health, and Safety Regulations for Effective PAM Adoption \\
\hline - Reframe perception of AM by overcoming throughput barrier \\
\hline Industry Collaborations to Enhance PAM Utility \\
\hline $\begin{array}{l}\text { - Pursue precision engineering and manufacturing } \\
\text { - Establish multi-stakeholder framework for coordinating R\&D in designing new materials } \\
\text { and processes specific for PAM } \\
\text { - Establish academic centers of research on simulations for PAM and develop projects with } \\
\text { industry and government labs } \\
\text { - Connect industry associations such as RadTech with stakeholders and the public for } \\
\text { deliberating the value of PAM }\end{array}$ \\
\hline
\end{tabular}




\section{Roadmapped Solutions}

To capture and consolidate the collective wisdom of the workshop panelists and participants, the committee generated Roadmapped Solutions, all of which fall into one of the five Recommended Research Directions (RRDs).

\section{Recommended Research Directions}

1. Material Science and Engineering to Characterize PAM Materials

2. Advanced Computing for Materials Discovery and Process Modelling

3. Measurement Science and Standards Development to Optimize PAM

4. Environmental, Health, and Safety Regulations for Effective PAM Adoption

5. Industry Collaborations to Enhance PAM Utility

Each Roadmapped Solution in the RRDs was further broken down into high, medium, and lower priority levels guided by the workshop transcripts. To aid understanding of the Roadmapped Solutions, the upper left corner of the map indicates the priority level while the upper right corner provides the abbreviated RRD to which they correspond. Each Roadmapped Solution is also comprised of eight categories:

- Objectives

- Describes the goals each solution is intended to serve

- Partners

○ Lists the stakeholder categories (i.e. Industry, Academic, Federal)

- Current Efforts

- Describes current progress in the field towards this solution

- Challenges

- Presents current barriers that could impair progress

- Goals timeline

- Breaks down actions into Near ( 0 to 3 years), Mid (4 to 6 years), and Long ( 7 years + ) term timelines

- Applications

- Provides examples for how and where this solution could be applied

- Risks

- Identifies Technical and Industrial risks with a High, Med, or Low rating

- Benefits

- Ranks benefits based on Economic, Productivity, Competitiveness categories with a High, Med, or Low designation 


\section{RRD 1: Material Science and Engineering to Characterize PAM Materials}

The Roadmapped Solutions under the Material Science and Engineering to Characterize PAM Materials Recommended Research Direction focus on the fundamental science needs in the field. The reliance on proprietary materials, testing and componentry limits fundamental scientific insight and the sharing of discovery between stakeholders. There must be standardized test geometries, resins, and light sources for development purposes. Test methods should leverage the process control inherent to PAM to build parametric datasets. New material classes, such as sustainable (e.g. bio-based, recyclable) resins can particularly benefit from standards. Listed below are each of the each roadmapped solutions with the corresponding panel in which they originated.

\section{High Priority Roadmapped Solutions:}

\section{Define properties of and relationships between bulk and printed materials}

- Probe the relationship between interlayer adhesion and final print strength to optimize final print properties

- Study dynamics of printing material in various forms: resin, pre-gelation, gelation, fully converted, and post processed

- Panel I: Sustainable, Hybrid, and Emerging Material Systems

Medium Priority Roadmapped Solutions:

Enhance in situ process control and monitoring with feedback

- Mature characterization technologies to understand material properties in the native printing environment which govern print resolution and minimum feature size

- Monitor mechanical and chemical properties during the printing process to inform feedback mechanisms to optimize final print

- Panel II: Novel, AM-specific characterization

Lower Priority Roadmapped Solutions:

Characterize dispersion and chemistry at nanoscale to inform part performance

- Probe the relationship between dispersion of resin components and final part performance

- Optimize filler dispersion and compatibility between fillers and polymer matrices to develop high performance parts

- Panel II: Novel, AM-specific characterization 


\section{Objectives}

- Properties of bulk materials that best predict properties of printed materials are determined

- Heterogeneity in final parts is predicted and incorporates into digital workflow

\begin{tabular}{|c|c|c|}
\hline & \multicolumn{2}{|c|}{ Partners } \\
\hline Industry (I) & Academic (A) & Federal (F) \\
\hline $\begin{array}{c}\text { Resin formulators, printer } \\
\text { OEMs, and end users }\end{array}$ & $\begin{array}{c}\text { Material scientists and } \\
\text { photochemistry specialists }\end{array}$ & $\begin{array}{c}\text { Measurement scientists and } \\
\text { material scientists }\end{array}$ \\
\hline
\end{tabular}

\section{Current Efforts (2020)}

- Bulk mechanical testing (I)

- Nanomechanical metrology(A\&F)

- Spectroscopy of printed parts (I, A, \& F)

\section{Challenges}

- Proprietary resins and hardware may respond different than models

- Digital design flexibility makes universal prediction difficult

- Applicability of research to manufacturing environment

\section{NEAR New tests to identify when bulk properties \\ (0-3 years)}

\section{Risks}

Scientific - Med

Engineering - High

Safety - High

Financial - Low
MID
$4-6$ years $)$

Develop tests and models that allow prediction of printed properties

(4-6 years)

\section{Benefits}

Economic- High

Productivity - High

EHS - Med
LONG
(7+years)
Penetrate markets that require specified material properties

\section{Applications}

Functionally engineered parts, tissue engineering, "meet the spec" to replace trad. manufacturing

ROADMAPPED SOLUTION: DEFINE PROPERTIES OF AND RELATIONSHIPS BETWEEN BULK AND PRINTED MATERIALS

Originating from PANel I: SUStainable, HyBRID, AND EMERging MATERIAl SYStems 


\title{
\begin{tabular}{|c|c|c|}
\hline Priority & Enhance In Situ Process Control and & RRD: Mat. Sci. \& \\
MEDIUM & Monitoring with Feedback & Materials \\
\hline
\end{tabular}
}

Objectives

- Develop tools to measure in situ properties and to generate feedback for quality control

- Demonstrate efficacy of in situ process modification

- Track defect formation and quality throughout print process

\begin{tabular}{|c|c|c|}
\hline \multicolumn{2}{|c|}{ Industry (I) } & Partners \\
\hline $\begin{array}{c}\text { Resin formulators, } \\
\text { printer OEMs, and end users }\end{array}$ & $\begin{array}{c}\text { Material scientists, analytical } \\
\text { chemists, and machine learning } \\
\text { experts }\end{array}$ & $\begin{array}{c}\text { R\&D agencies and open-user } \\
\text { facilities }\end{array}$ \\
\hline
\end{tabular}

Current Efforts (2020)

- Interferometry and force microscopy in situ measurements (A \& F)

- Thermography (I, A, \& F)

- Closed-loop control systems for PAM (A)

\section{Challenges}

- Lack of tools with needed capacity

- Complexity of processes, technology to integrate control, spatial resolution, 3D needs

- Multiple skill sets required

- Pre-competitive openness

\section{NEAR Develop tools to probe properties (0-3 years) throughout printing}

\section{Risks \\ Scientific - High \\ Engineering - High \\ Safety - Low \\ Financial - Med}

$$
\begin{array}{r|r}
\text { MID } & \text { Incorporate data acquisition to enable } \\
\text { (4-6 years) } & \text { rapid feedback and process control }
\end{array}
$$

\section{Benefits \\ Economic- High \\ Productivity - High \\ EHS - Low}

\begin{abstract}
Applications
Manufacturing processes across the supply chain, analytical instrument development
\end{abstract}

ROADMAPPED SOLUTION: ENHANCE IN SITU PROCESS CONTROL AND MONITORING WITH FEEDBACK

Originating from PANel II: NOVEL, AM-SPECIFIC CHARACTERIZATION 


\section{Objectives}

- Integrate dispersion and interface characterization into performance metrics

- Link resin material properties and performance to specific printing technologies

\begin{tabular}{|c|c|c|}
\hline & Partners & \\
\hline Industry (I) & Academic (A) & Federal (F) \\
\hline $\begin{array}{c}\text { Resin formulators, } \\
\text { printer OEMs, and end users }\end{array}$ & $\begin{array}{c}\text { Material scientists, analytical } \\
\text { chemists, and data scientists }\end{array}$ & $\begin{array}{c}\text { R\&D agencies and open-user } \\
\text { facilities }\end{array}$ \\
\hline
\end{tabular}

\section{Current Efforts (2020)}

- Scatterometry (A)

- Chemical spectroscopy (I, A, \& F)

- Optical microscopy (A \& F)

- Mechanical property testing via scanning probe microscopy, nanoindentation, tensile/compression testing (I, A, \& F)

\section{Challenges}

- Multi-phase characterizations

- Complexity

- Different types of measurands (chemical, physical)

- Multiple skill sets required

- Pre-competitive openness

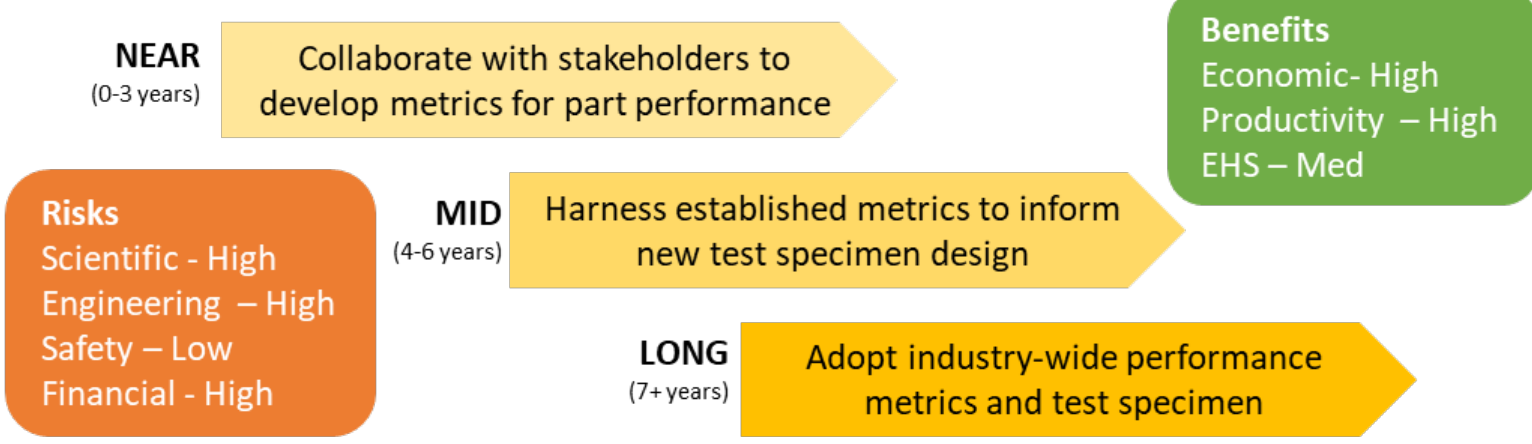

\section{Applications}

Composite material development, tissue engineering

ROADMAPPED SOLUTION: CHARACTERIZE DISPERSION AND CHEMISTRY AT NANOSCALE TO INFORM PART PERFORMANCE

OrIGINATING FROM PANEL II: NOVEL, AM-SPECIFIC CHARACTERIZATION 


\section{RRD 2: Advanced Computing for Materials Discovery and Process Modelling}

The Roadmapped Solutions under the Advanced Computing for Materials Discovery and Process Modelling Recommended Research Direction focus on the requisite computational needs to expedite new material development and optimize printing. As a digital manufacturing technology, PAM must leverage advanced computational methods to discover new materials and ensure their optimal processability. Computational methods ranging from machine learning to multi-scale modeling can be used to simultaneously optimize cost, throughput and performance of new materials and processes. The development of robust databases is essential in inform and validate computational approaches. Listed below are each of the each roadmapped solutions with the corresponding panel in which they originated.

High Priority Roadmapped Solutions:

Build a model to correlate printing conditions and final properties

- Final print properties determine utility of PAM technology and a model of printing process will inform ways to increase throughput while maintaining print quality

- Panel II: Novel, AM-specific characterization

Launch major efforts in computing and informatics to predict print process

- Harness large amounts of data generated by printing process to characterize and optimize PAM for a given resin and printing condition facilitated by advances in computing capacity

- Panel V: Moonshot Ideas in PAM

Medium Priority Roadmapped Solutions:

Develop empirically-informed theory, modeling, and simulations of PAM

- To fully engineer the functionality of PAM parts and increase print throughput, comprehensive theory, modeling, and simulations of the printing process is required

- Panel IV: Health, Safety and Regulation in AM

Improve computational models for UV-resin interactions

- Models informing UV-resin interactions will facilitate increased build efficiency

- Panel I: Sustainable, Hybrid, and Emerging Material Systems

Lower Priority Roadmapped Solutions:

Create database of existing renewable photopolymers

- Develop knowledge hubs on current understanding of reinforcements and additives to expedite high performance, renewable materials for PAM

- Create (or centralize existing) toxicity databases will decrease confusion regrading safety protocols and expedite PAM adoption

- Panel I: Sustainable, Hybrid, and Emerging Material Systems 


\section{Objectives}

- $\quad$ Build tools to predict printing processes and material properties

- $\quad$ Predict performance before parts are printed with a given PAM technique and material

- Develop shared resources

\begin{tabular}{|c|c|c|c|}
\hline \multicolumn{4}{|c|}{ Partners } \\
\hline Industry (I) & \multicolumn{2}{|c|}{ Academic (A) } & Federal (F) \\
\hline $\begin{array}{l}\text { Resin formulators, } \\
\text { printer OEMs, and end users }\end{array}$ & \multicolumn{2}{|c|}{$\begin{array}{l}\text { Material scientists, polymer } \\
\text { chemists, and data scientists }\end{array}$} & $\begin{array}{c}\text { R\&D agencies, manufacturing } \\
\text { programs, and statisticians }\end{array}$ \\
\hline \multicolumn{2}{|c|}{$\begin{array}{l}\text { Current Efforts (2020) } \\
\text { - Empirical model development (I, A, \& F) } \\
\text { - } \text { First principle models (A \& F) } \\
\text { - } \text { Print environ. characterization (I, A, \& F } \\
\text { - Process visualization and analytics to } \\
\text { predict part properties (A) }\end{array}$} & $\begin{array}{l}\text { - Engagem } \\
\text { commerc } \\
\text { - Time to c } \\
\text { - Industry } \\
\text { manufac } \\
\text { samples }\end{array}$ & $\begin{array}{l}\text { Challenges } \\
\text { nt of model developers, } \\
\text { al package developers } \\
\text { velop models and test data } \\
\text { buy in and ability to } \\
\text { are and characterize test }\end{array}$ \\
\hline
\end{tabular}

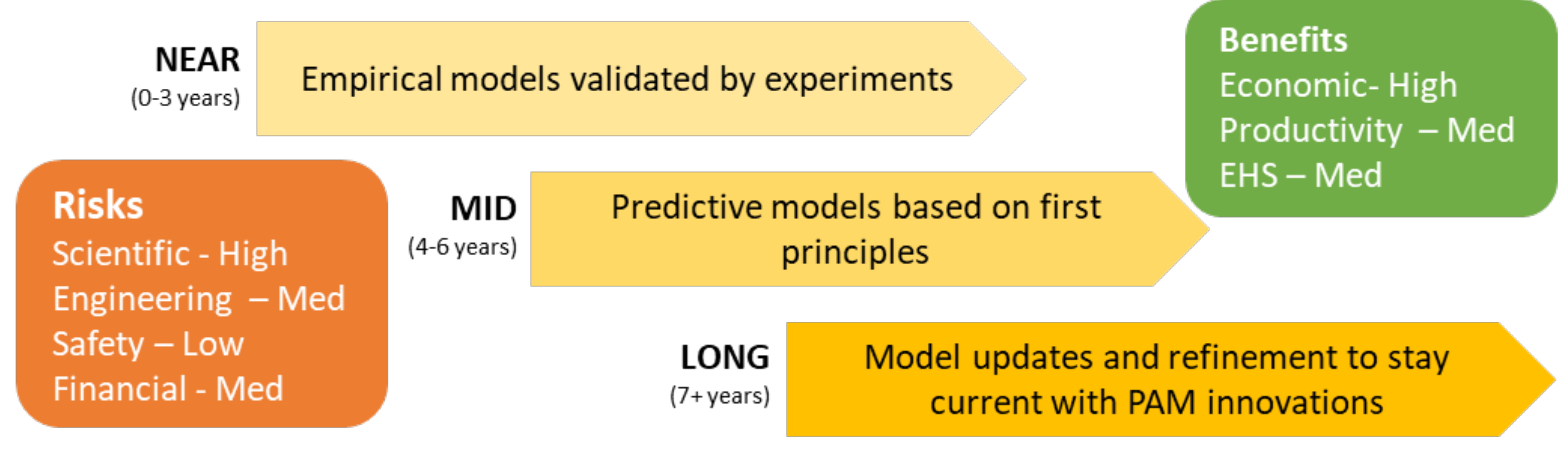

\section{Applications}

Functionally engineered parts, personalized medicine, optimized PAM throughput

ROADMAPPED SOLUTION: BUILD A MODEL TO CORRELATE PRINTING CONDITIONS AND FINAL PROPERTIES

ORIGINATING fROM PANel II: NOVEL, AM-SPECIFIC CHARACTERIZATION 


\section{Launch Major Efforts in Computing and Informatics to Predict Print Process}

RRD: Adv. Comp. for Mat. Disc \& Process Modelling

\section{Objectives}

- Integrate effort among academia, industry, and government

- Enhance prediction capabilities for materials, defects, safety

- Grow U.S. expertise in PAM technologies

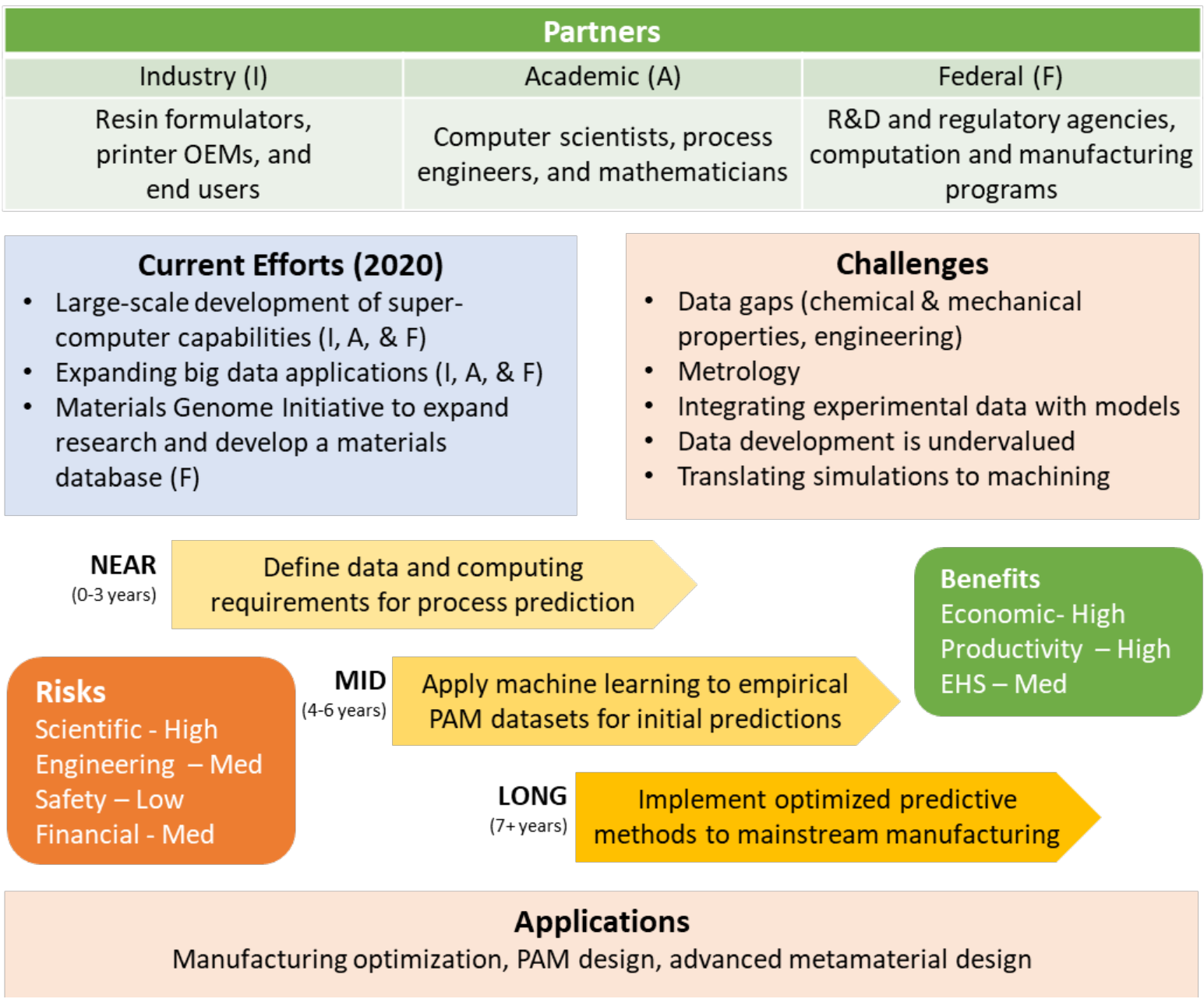

ROADMAPPED SOLUTION: LAUNCH MAJOR EFFORTS IN COMPUTING AND INFORMATICS TO PREDICT PRINT PROCESS ORIGINATING FROM PANEL V: MOONSHOt IDEAS IN PAM 


\section{$\begin{array}{ccc}\begin{array}{c}\text { Priority } \\ \text { MEDIUM }\end{array} & \begin{array}{c}\text { Develop Empirically-informed Theory, } \\ \text { Modeling, and Simulations of PAM }\end{array} & \begin{array}{c}\text { RRD: Adv. Comp. } \\ \text { for Mat. Disc \& } \\ \text { Process Modelling }\end{array}\end{array}$}

\section{Objectives}

- Develop robust tools to enable production safety

- Build scientific basis for materials design and life cycle analysis

- Combine modeling and simulation capabilities with experimental theory capabilities

\begin{tabular}{|c|c|c|}
\hline \multicolumn{2}{|c|}{ Partners } \\
\hline Industry (I) & Academic (A) & Federal (F) \\
\hline $\begin{array}{c}\text { Resin formulators and } \\
\text { printer OEMs }\end{array}$ & $\begin{array}{c}\text { Material scientists, chemists, } \\
\text { and computer scientists }\end{array}$ & $\begin{array}{c}\text { R\&D agencies, computation and } \\
\text { data centers }\end{array}$ \\
\hline
\end{tabular}

\section{Current Efforts (2020)}

- Searchable repositories of experiments and data (I)

- Advanced visualizations of data to emphasize parameter correlations (I)

- Calculations to predict process parametersprint property relationships (I, A, \& F)

\section{Challenges}

- Lack of software for design, control and modeling

- Linking simulation platforms to physical and chemical property databases

- Interpreting results and applying to the field

\section{NEAR \\ (0-3 years)

$$
\begin{gathered}
\text { Develop experimental basis for } \\
\text { computational input }
\end{gathered}
$$

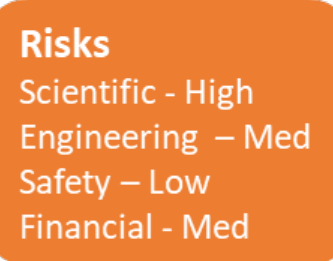

MID Imp Implement process-level models and
simulations

\section{Benefits}

Economic- Med

Productivity - High EHS - Med

\section{Applications}

PAM product design, PAM process optimization, resin formulation

ROADMAPPED SOLUTION: DEVELOP EMPIRICALLY-INFORMED THEORY, MODELING, AND SIMULATIONS OF PAM

Originating from Panel IV: Health, Safety and Regulation in AM 


\section{Priority
Improve Computational Models for UV-Resin
Interactions}

RRD: Adv. Comp. for Mat. Disc \& Process Modelling

\section{Objectives}

- Identify models, tools, and available data

- Generate new data to dynamically enhance prediction and capabilities

- Harmonize outputs with tool kits and validation

\begin{tabular}{|c|c|c|}
\hline & Partners \\
\hline Industry (I) & Academic (A) & Federal (F) \\
\hline $\begin{array}{c}\text { Resin formulators, } \\
\text { printer OEMs, and } \\
\text { trade organizations }\end{array}$ & $\begin{array}{c}\text { Computational and modelling } \\
\text { specialists }\end{array}$ & $\begin{array}{c}\text { Database and material } \\
\text { standards experts }\end{array}$ \\
\hline
\end{tabular}

\section{Current Efforts (2020)}

- UV cured film characterization (I, A, \& F)

- $2 D$, thin film approximations applied to single-layer properties (I, A, \& F)

- Bulk material approximations applied to fully printed parts (I, A, \& F)

\section{Challenges}

- Collaborative computational model platforms with input from industry

- Variable scaling, complexity

- Linking advances in measurements to input data \& design

- Keeping models current

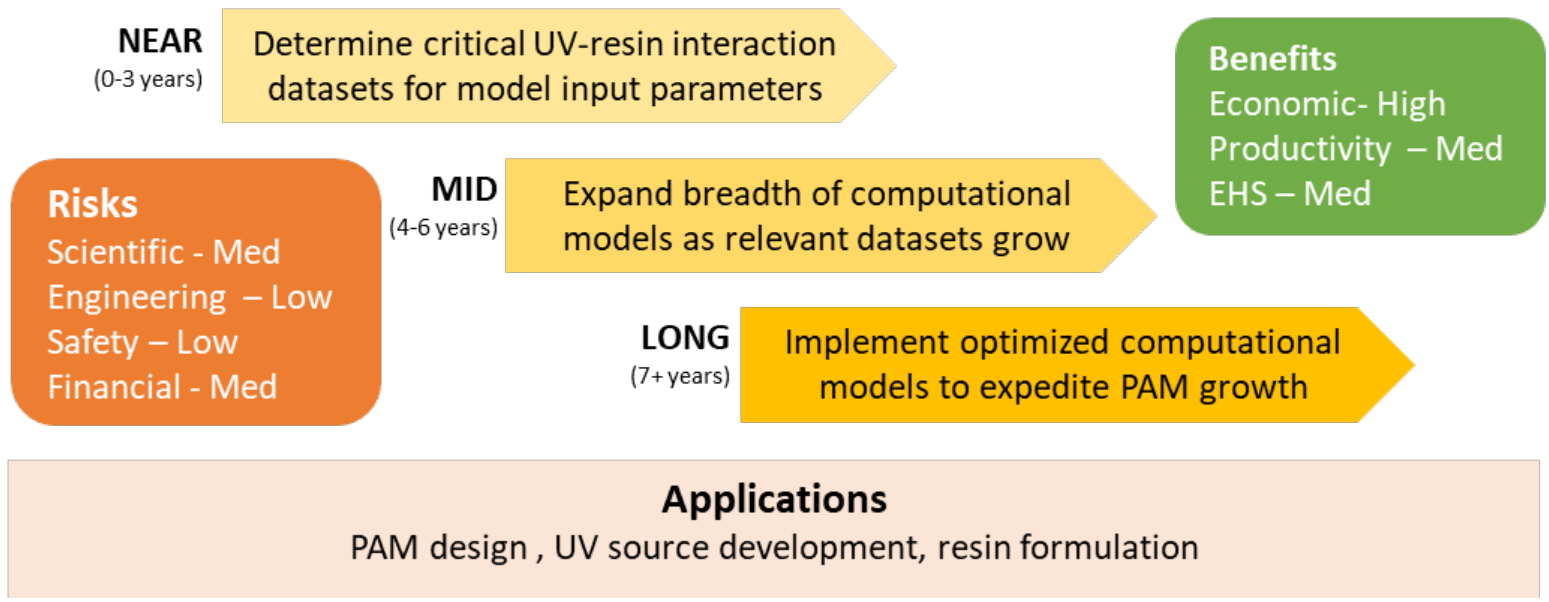

ROADMAPPED SOLUTION: IMPROVE COMPUTATIONAL MODELS FOR UV-RESIN INTERACTIONS

Originating from Panel I: Sustainable, Hybrid, AND EMERging Material Systems 


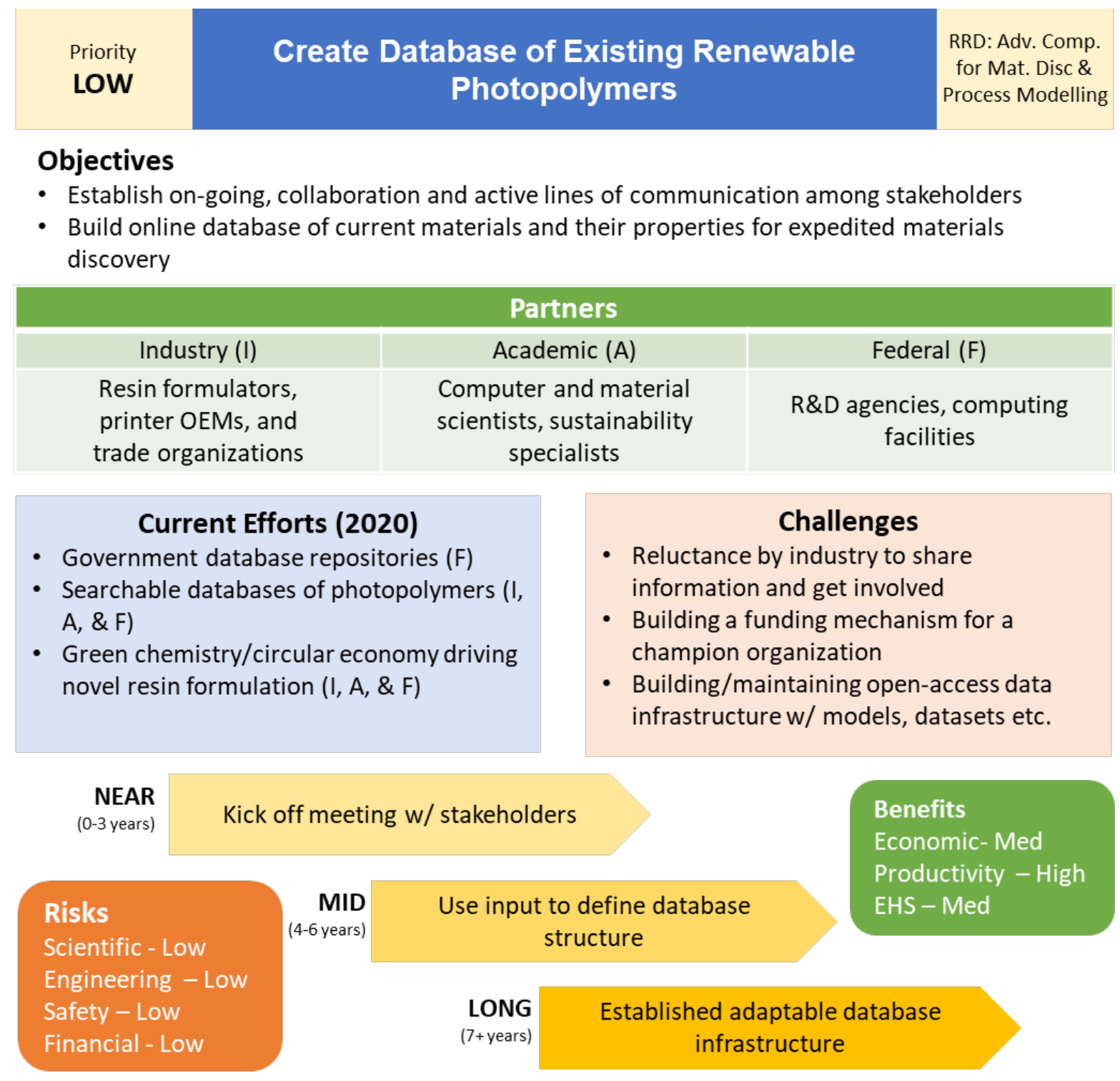

\section{Applications}

R\&D efforts across academia, industry, and federal agencies

RoAdmapped SOlution: Create datABASE Of EXISTING ReNeWAble Photopolymers

Originating from Panel I: Sustainable, Hybrid, ANd Emerging Material Systems 


\section{RRD 3: Measurement Science and Standards Development to Optimize PAM}

The Roadmapped Solutions under the Measurement Science and Standards Development to Optimize PAM focus on the metrology and standards needed for PAM growth. The reliance on proprietary materials, testing and componentry limits fundamental scientific insight and the sharing of discovery between stakeholders. There must be standardized test geometries, resins, and light sources for development purposes. Test methods should leverage the process control inherent to PAM to build parametric datasets. New material classes, such as sustainable (e.g. bio-based, recyclable) resins can particularly benefit from standards. Listed below are each of the each roadmapped solutions with the corresponding panel in which they originated.

High Priority Roadmapped Solutions:

Develop PAM-specific standards and calibrations

- Standards and calibrations development for both PAM resins and the PAM process expedite the optimization of printed parts while making technology more accessible to new applications

- Panel II: Novel, AM-specific characterization

Advance measurement science and tools to optimize quality control

- Quality of PAM parts must be optimized for a given applications and investments in versatile instruments to probe a variety of resins and print conditions is necessary

- Panel II: Novel, AM-specific characterization

Medium Priority Roadmapped Solutions:

Develop resin standards for sustainability market needs

- Sustainable resin market requires definition and implementation of standards for successful adoption

- Panel I: Sustainable, Hybrid, and Emerging Material Systems

Optimize UV sources for PAM Applications

- Determining the most effective and efficient photopolymerization sources critical to process control

- Panel I: Sustainable, Hybrid, and Emerging Material Systems

Lower Priority Roadmapped Solutions:

Expedite material characterization via machine learning

- Use data generated by experimental PAM to inform machine learning algorithms designed to characterize a given material

- Panel V: Moonshot Ideas in PAM 


\section{Objectives}

- Develop validated and commonly accepted sets of test methods for material properties

- Grow measurement capabilities over wide temporal and spatial scale ranges

- Develop quality data for regulations

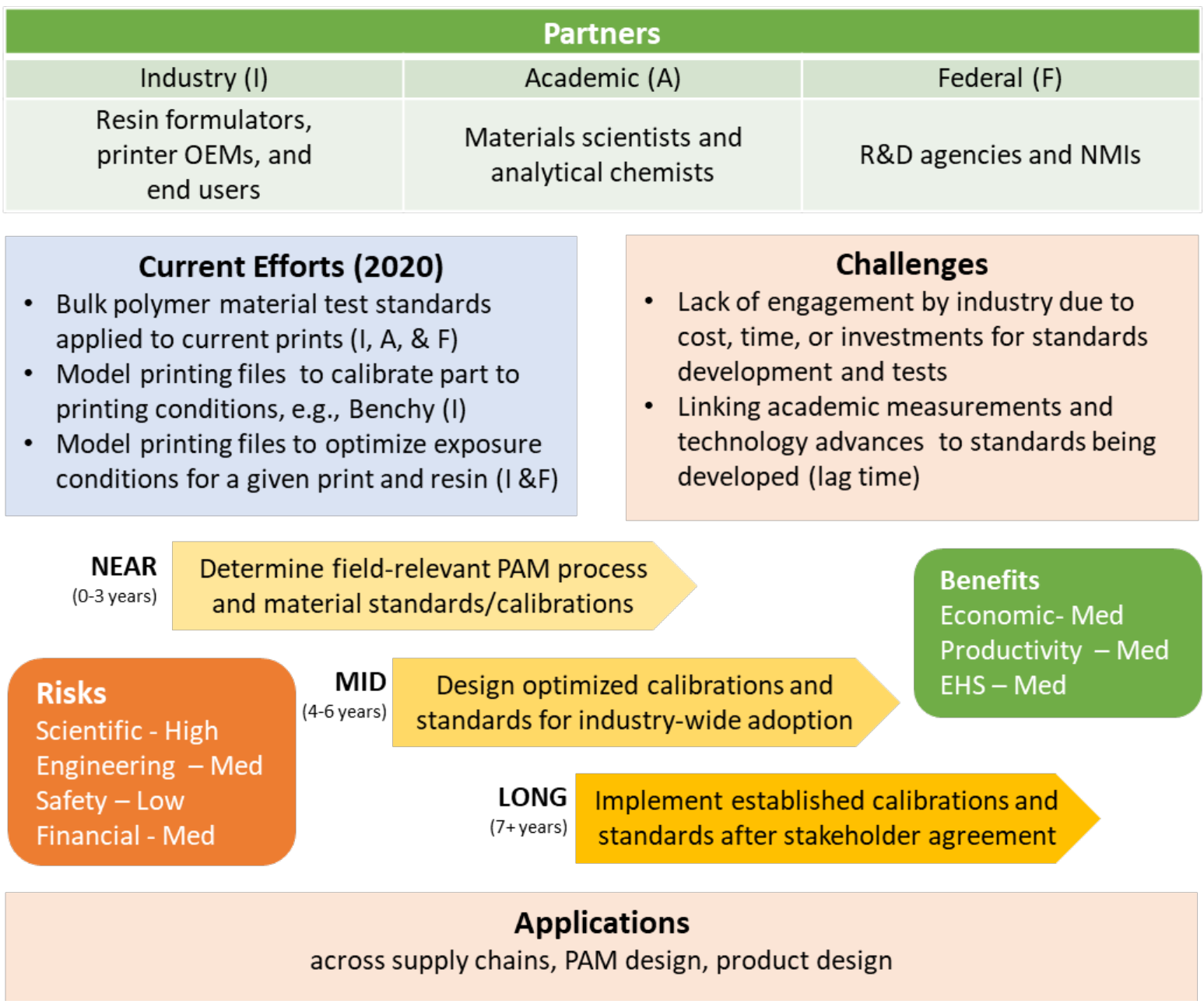

ROADMAPPED SOLUTION: DEVELOP PAM-SPECIFIC STANDARDS AND CALIBRATIONS

ORIGINATING FROM PANEL II: NOVEL, AM-SPECIFIC CHARACTERIZATION 


\section{Objectives}

- Develop deployable tools with relevant sensitivity, resolution, and precision

- Improve quality control and consistency for manufacturing applications

- Reduce trial and errors in R\&D time

\begin{tabular}{|c|c|c|}
\hline & \multicolumn{2}{|c|}{ Partners } \\
\hline Industry (I) & Academic (A) & Federal (F) \\
\hline $\begin{array}{c}\text { Resin formulators, } \\
\text { printer OEMs, and end users }\end{array}$ & $\begin{array}{c}\text { Material scientists, analytical } \\
\text { chemists, and metrology } \\
\text { experts }\end{array}$ & R\&D and regulatory agencies \\
\hline
\end{tabular}

\section{Current Efforts (2020)}

- Optical metrology to inspect part for defects (I, A, \& F)

- Print green strength calibration to optimize part fidelity (I)

- Spectroscopy on resin (I, A, \& F)

- Bulk mechanical testing (e.g.,dog-bone) (I)

\section{Challenges}

- Lack of universally accepted metrics

- Linking R\&D successes to adoptable realtime process integration

- Disconnect between characterization speed and high throughput PAM

- Rapid new material developments

\section{NEAR \\ (0-3 years) \\ Establish appropriate metrics and tools to optimize part quality}

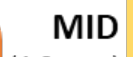
(4-6 years)

Develop tools to capture relevant metrics for quality control
Benefits

Economic- High

Productivity - High

EHS - Low

Scientific - High

Engineering - High

Safety - Low

Financial - Med
LONG
(7+years)
Equip PAM stakeholders with tools to optimize quality control

\section{Applications}

Materials development, manufacturing and product production

ROADMAPPED SOLUTION: ADVANCE MEASUREMENT SCIENCE AND TOOLS TO OPTIMIZE QUALITY CONTROL

Originating fRom PANel II: NOVEL, AM-SPECIFIC ChARACTERIZATION 


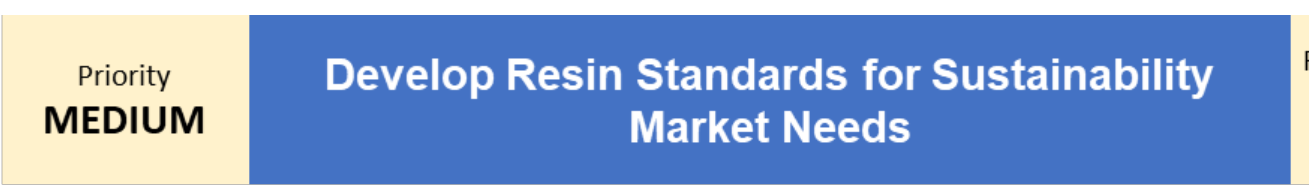

RRD: Meas. Sci. \& Stds. Dev. to Optimize PAM

\section{Objectives}

- Establish baseline physical and chemical characteristics to define sustainable or renewable aspects of products

- Identify and address problem areas that limit the use of new resin materials

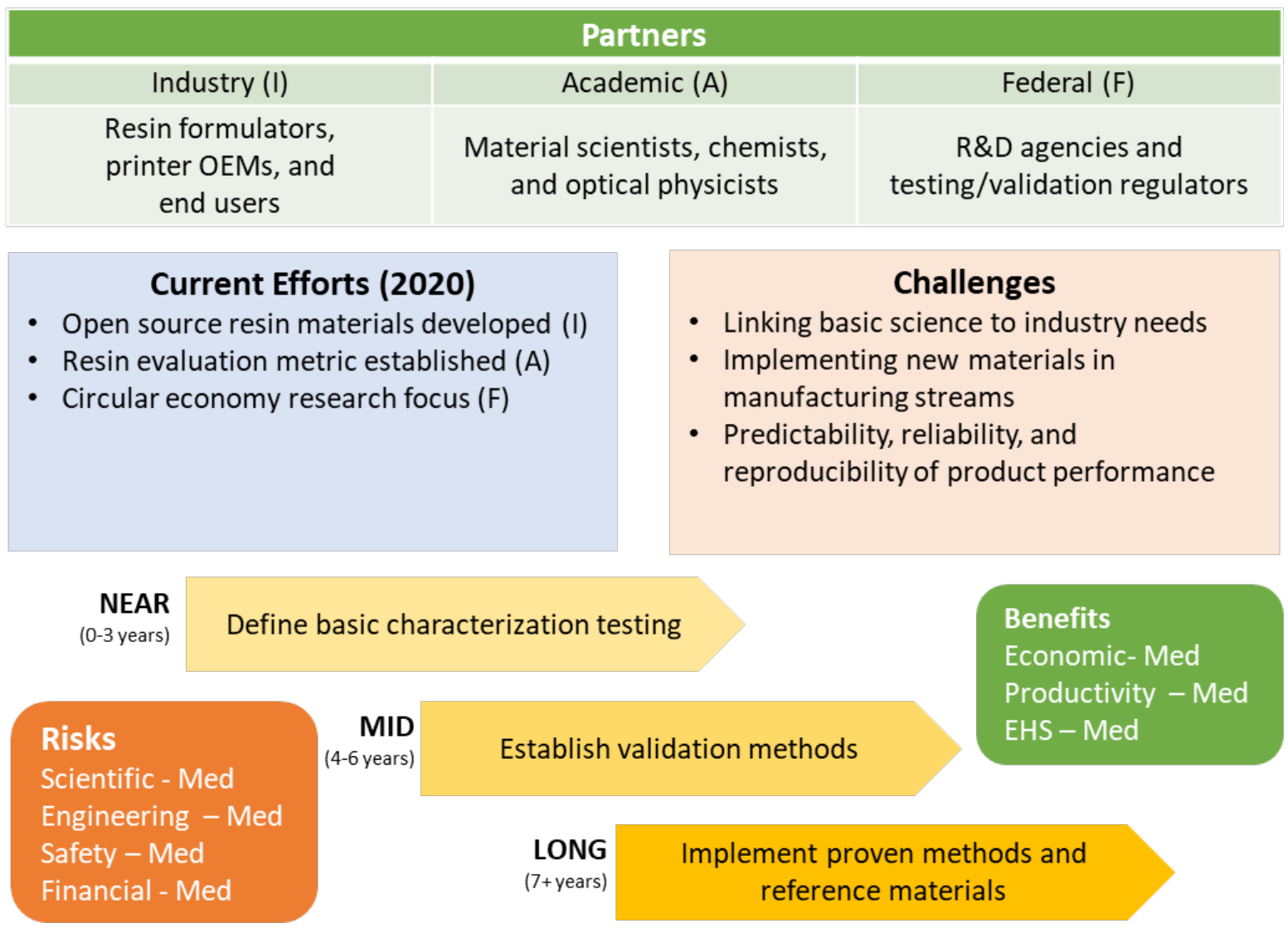

\section{Applications}

Design of new materials from fundamental science input

ROADMAPPED SOLUTION: DEVELOP RESIN STANDARDS FOR SUSTAINABILITY MARKET NEEDS

Originating from PANel I: Sustainable, Hybrid, AND Emerging Material Systems 


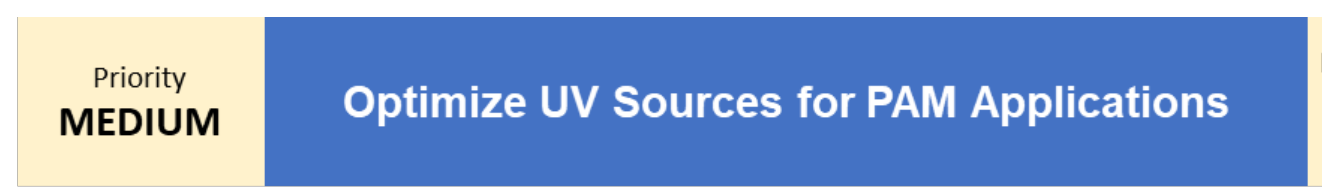

RRD: Meas. Sci. \&

Stds. Dev. to

Optimize PAM

\section{Objectives}

- Optimize UV exposure conditions for each PAM system and material

- $\quad$ Establish guidelines to meet equipment specifications by engaging printer OEMs

- $\quad$ Facilitate implementation of new light-engine technologies

\begin{tabular}{|c|c|c|}
\hline \multicolumn{3}{|c|}{ Partners } \\
\hline Industry (I) & Academic (A) & Federal (F) \\
\hline $\begin{array}{c}\text { Resin formulators, } \\
\text { printer OEMs, and } \\
\text { UV source manufacturers }\end{array}$ & $\begin{array}{c}\text { Optical physicists, mechanical } \\
\text { and optical engineers }\end{array}$ & $\begin{array}{c}\text { R\&D agencies specializing in } \\
\text { optics and material science }\end{array}$ \\
\hline
\end{tabular}

\section{Current Efforts (2020)}

- Thin film UV cure characterization (I)

- Metrologically characterized UV source (I \& F)

- Emissive display characterization and production (I, A, \& F)

\section{Challenges}

- Industry -wide changes to equipment

- Multi-scale (batch vs. high volume lamps)

- PAM UV niche vs. big consumables markets for lamp industry

- Time-warp in photochemistry R\&D and academic skill sets and focus

\section{NEAR \\ (0-3 years) \\ Explore new light-engine technologies for specific PAM applications}

Benefits

Economic- Med

Productivity - Med

\section{EHS - Low \\ Risks MID Characterize and optimize UV light \\ Scientific-Med (4-6years) engines that enable high-throughput \\ Engineering - Med \\ Safety - Low \\ Financial - Med
LONG
(7+years)
Implement optimized UV sources into
production lines with feedback control

\section{Applications}

Equipment manufacturers, UV industry, material curing

ROAdMAPPED SOlution: Optimize UV SOURCES FOR PAM APPLICATIONS

Originating from PAnel I: Sustainable, Hybrid, ANd Emerging MAterial Systems 


\section{Expedite Material Characterization via Machine \\ Learning}

RRD: Meas. Sci. \&

Stds. Dev. to

Optimize PAM

\section{Objectives}

- Improve communication of PAM needs and progress through the value chain

- Enhance use of data while establishing cybersecurity framework for shared data

- Increase data value for PAM applications via machine learning (ML)

\begin{tabular}{|c|c|c|c|}
\hline \multicolumn{4}{|c|}{ Partners } \\
\hline Industry (I) & \multicolumn{2}{|c|}{ Academic (A) } & Federal (F) \\
\hline $\begin{array}{c}\text { Resin formulators, } \\
\text { printer OEMs, trade } \\
\text { organizations, and end users }\end{array}$ & \multicolumn{2}{|c|}{$\begin{array}{l}\text { Data science, cybersecurity and } \\
\text { manufacturing specialists }\end{array}$} & $\begin{array}{c}\text { Manufacturing programs, } \\
\text { cybersecurity and data analytics } \\
\text { experts }\end{array}$ \\
\hline $\begin{array}{l}\text { Current Efforts (2 } \\
\text { - Machine learning applicatio } \\
\text { development (I, A, \& F) } \\
\text { - Materials genome initiative } \\
\text { - } \quad \text { Machand new classes of mate } \\
\text { quality assessment/predicti }\end{array}$ & $\begin{array}{l}\text { 20) } \\
\text { to materials } \\
\text { identify and } \\
\text { als (F) } \\
\text { letection and } \\
\text { (I \& F) }\end{array}$ & $\begin{array}{l}\text { - Hobbyist } \mathrm{p} \\
\text { applicatio } \\
\text { - Perceived } \\
\text { - Lacking cy } \\
\text { threats } \\
\text { - Value chai }\end{array}$ & $\begin{array}{l}\text { Challenges } \\
\text { perception of technology } \\
\text { ns } \\
\text { value of data low } \\
\text { bersecurity understanding and } \\
\text { n buy-in }\end{array}$ \\
\hline
\end{tabular}

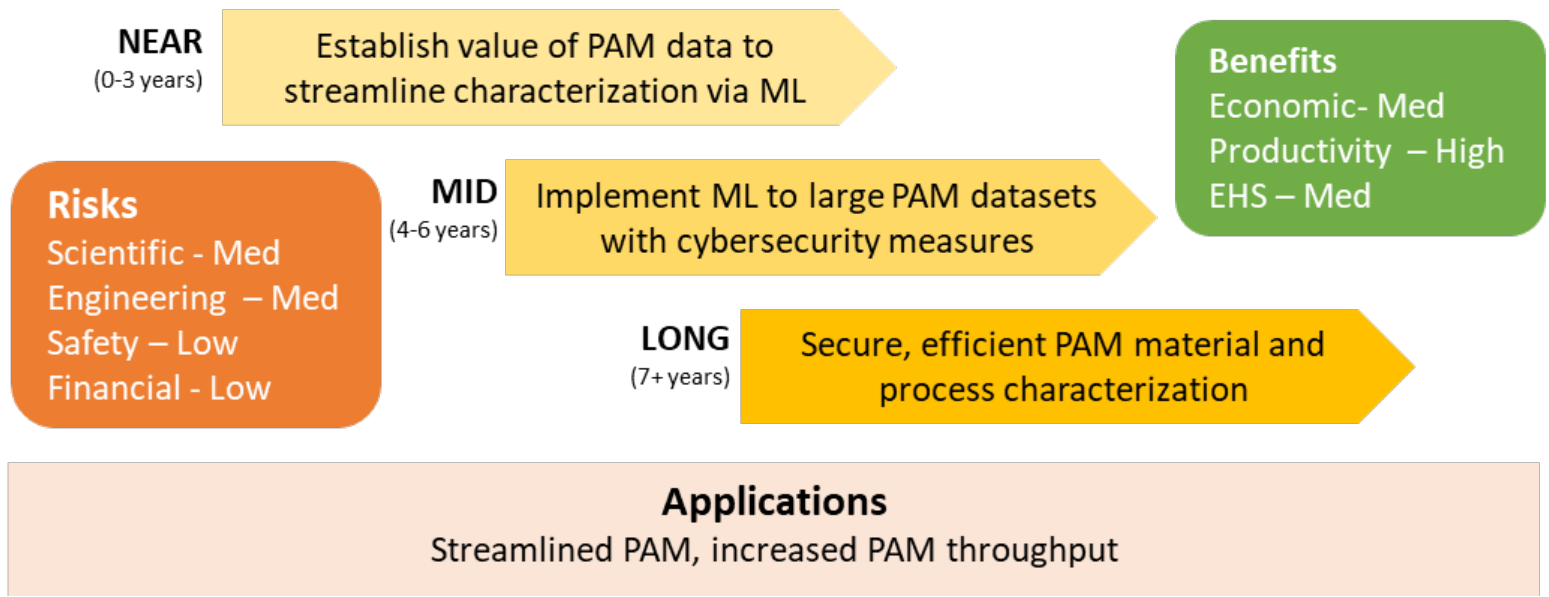

ROADMAPPED SOLUTION: EXPEDITE MATERIAL CHARACTERIZATION VIA MACHINE LEARNING

ORIGINATING FROM PANEL V: MOONSHOT IDEAS IN PAM 


\section{RRD 4: Environmental, Health, and Safety Regulations for Effective PAM Adoption}

The Roadmapped Solutions under the Environmental, Health, and Safety Regulations for Effective PAM Adoption Recommended Research Direction focus on the EHS regulations essential to guiding the industry. PAM technology adoption has spread rapidly in recent years, catching regulatory agencies by surprise. Given the unique handling requirements of PAM resins and post-process needs of PAM parts, specific data, guidelines and education for user and environmental safety are needed. Listed below are each of the each roadmapped solutions with the corresponding panel in which they originated.

High Priority Roadmapped Solutions:

Support data systems for PAM registries and regulations

- Engage proactively with regulators and legislators in setting standards for new materials registrations

- Industry associations inform and engage with safety and regulations continuously and provide information sessions at meetings, workshops etc.

- Panel III: Industry Applications and Developing Markets

Reframe perception of PAM by overcoming throughput barrier

- Create awareness for utilities of PAM in industries requiring customization while increasing throughput by optimizing the print process

- Panel V: Moonshot Ideas in PAM

Medium Priority Roadmapped Solutions:

Define minimal dataset for risk assessment, exposure

- Adopt approaches and methods from other industries, such as nanotechnology, or develop new methods for streamlining risk assessment

- Panel IV: Health, Safety and Regulation in AM

Develop framework for product stewardship

- Build safety concepts into product launch models and business plans

- Collect, review, and disseminate current and best practices to enhance life cycle analysis

- Panel IV: Health, Safety and Regulation in AM

Lower Priority Roadmapped Solutions:

\section{Design education material on green photopolymers}

- Facilitate adoption of green photopolymers by communicating with stakeholders the utility of sustainable materials for use in PAM

- Panel I: Sustainable, Hybrid, and Emerging Material Systems 


\section{Support Data Systems for PAM Registries and Regulations}

RRD: EHS Reg. for

Effective PAM Adoption

\section{Objectives}

- Enhance access to data systems for regulation development with regulatory agencies

- Establish help lines with direct links to regulatory offices

\begin{tabular}{|c|c|c|}
\hline \multicolumn{3}{|c|}{ Partners } \\
\hline Industry (I) & Academic (A) & Federal (F) \\
\hline $\begin{array}{l}\text { Resin formulators, } \\
\text { printer OEMs, and } \\
\text { trade organizations }\end{array}$ & Data scientists & $\begin{array}{l}\text { Database experts and } \\
\text { regulatory agencies }\end{array}$ \\
\hline
\end{tabular}

\section{Current Efforts (2020)}

- Photopolymer databases (I \& F)

- Resin material approval process in development (F)

- Toxicity evaluation procedures (F)

- Application of regulation approach with previous novel materials to PAM (F)

\section{Challenges}

- Understanding what data are needed

- Pre-market data use

- Education and training on new tools

- Awareness of PAM in existing products and capabilities

- Lack of tools and starting from scratch
NEAR
(0-3 years)

$$
\begin{aligned}
& \text { Engage all PAM stakeholder to } \\
& \text { establish regulation needs }
\end{aligned}
$$

\section{Risks \\ Scientific - Med \\ Engineering - Med \\ Safety - Low \\ Financial - Med}

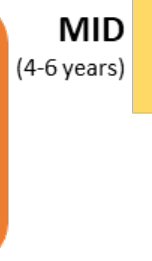
Develop platforms for agencies to implement regulations

\section{Benefits}

Economic- Med

Productivity - Med

EHS - High

\section{LONG Streamline process for regulations to be maintained and satisfied}

\section{Applications}

PAM quality control, enhancing market-wide PAM adoption

ROADMAPPED SOLUTION: SUPPORT DATA SYSTEMS FOR PAM REGISTRIES AND REGULATIONS

ORIGINATING FRom PANEL III: INDUSTRY APPLICATIONS AND DEVELOPINg MARKETS 


\begin{tabular}{|c|c|}
\hline Priority & Reframe Perception of AM by Overcoming \\
HIGH & $\begin{array}{c}\text { RRD: EHS Reg. for } \\
\text { Effective PAM } \\
\text { Adoption }\end{array}$ \\
\hline
\end{tabular}

\section{Objectives}

- Enhance PAM throughput capabilities via multi-stakeholder collaboration

- Launch efforts to highlight PAM throughput advancements

\begin{tabular}{|c|c|c|}
\hline & Partners & Federal (F) \\
\hline Industry (I) & Academic (A) & Database and material \\
\hline $\begin{array}{l}\text { Marketing and sales specialists, } \\
\text { resin formulators, printer OEMs }\end{array}$ & $\begin{array}{c}\text { Material scientists, PAM and } \\
\text { supply chain specialists }\end{array}$ & \begin{tabular}{c} 
standards experts \\
\hline
\end{tabular} \\
\hline
\end{tabular}

\section{Current Efforts (2020)}

- Large-format printing PAM technologies being brought to market (I \& A)

- Research to increase speed and throughput of printing (A \& F)

- Workshops to engage PAM stakeholders to discuss throughput advances (I, A, \& F)

\section{Challenges}

- Large-scale PAM part performance not validated

- Fast chemistries require control of exothermic heat which affect throughput

- Potential costly economic investment to achieve competitive throughput

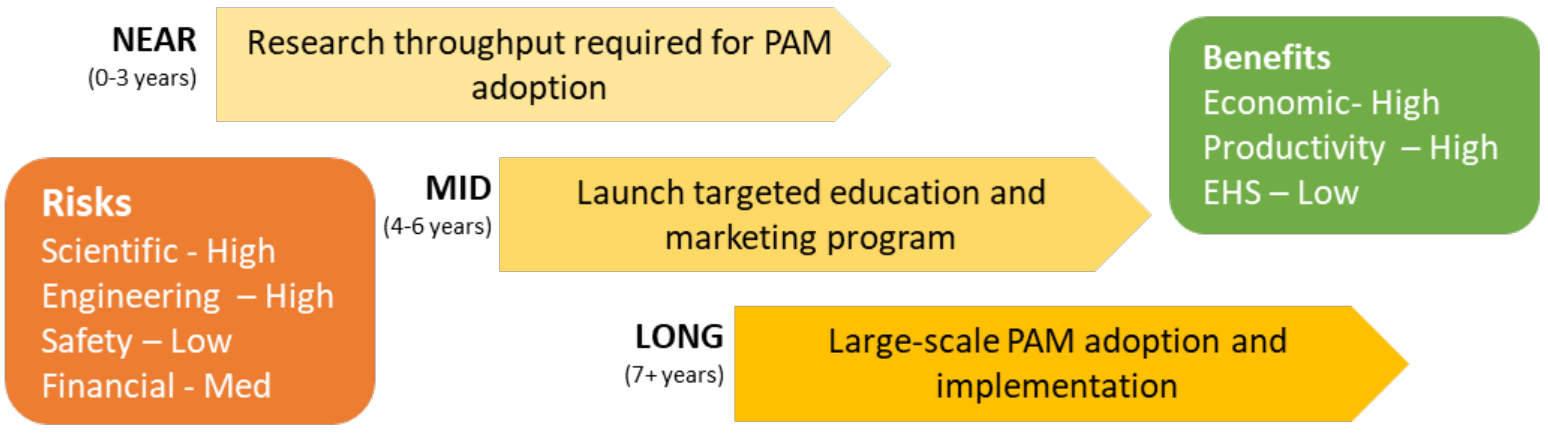

\section{Applications}

Personalized medicine, engineered metamaterials, automotive and aerospace implementation

ROADMAPPED SOLUTION: REFRAME PERCEPTION OF PAM BY OVERCOMING THROUGHPUT BARRIER

ORIGINATING FRom PANel V: MoONShot IDEAS IN PAM 


\section{Priority
MEDIUM}

RRD: EHS Reg. for

Effective PAM

Adoption

\section{Objectives}

- Harmonize toxicological evaluations with exposure and risk assessments for new materials and products

- Establish guidelines to define minimal data requirements for new materials and products

\begin{tabular}{|c|c|c|}
\hline & \multicolumn{2}{|c|}{ Partners } \\
\hline $\begin{array}{c}\text { Industry (I) } \\
\text { Resin formulators, } \\
\text { printer OEMs, trade } \\
\text { organizations, and end users }\end{array}$ & $\begin{array}{c}\text { Academic (A) } \\
\text { Toxicologists and environmental } \\
\text { engineers }\end{array}$ & $\begin{array}{c}\text { R\&D and regulatory agencies, } \\
\text { international entities }\end{array}$ \\
\hline
\end{tabular}

\section{Current Efforts (2020)}

- Baseline toxicity levels for volatile organic compounds established (A \& F)

- In situ and in vivo PAM biocompatibility studies for various materials ( I, A, \& F)

- Baseline resin exposure levels established for some resins (F)

\section{Challenges}

- Consensus building

- Multi-disciplinary expertise required in toxicology, exposure, and risk assessment

- Experimental data requirements

- Existing data gaps extensive and will require substantial resources to fill

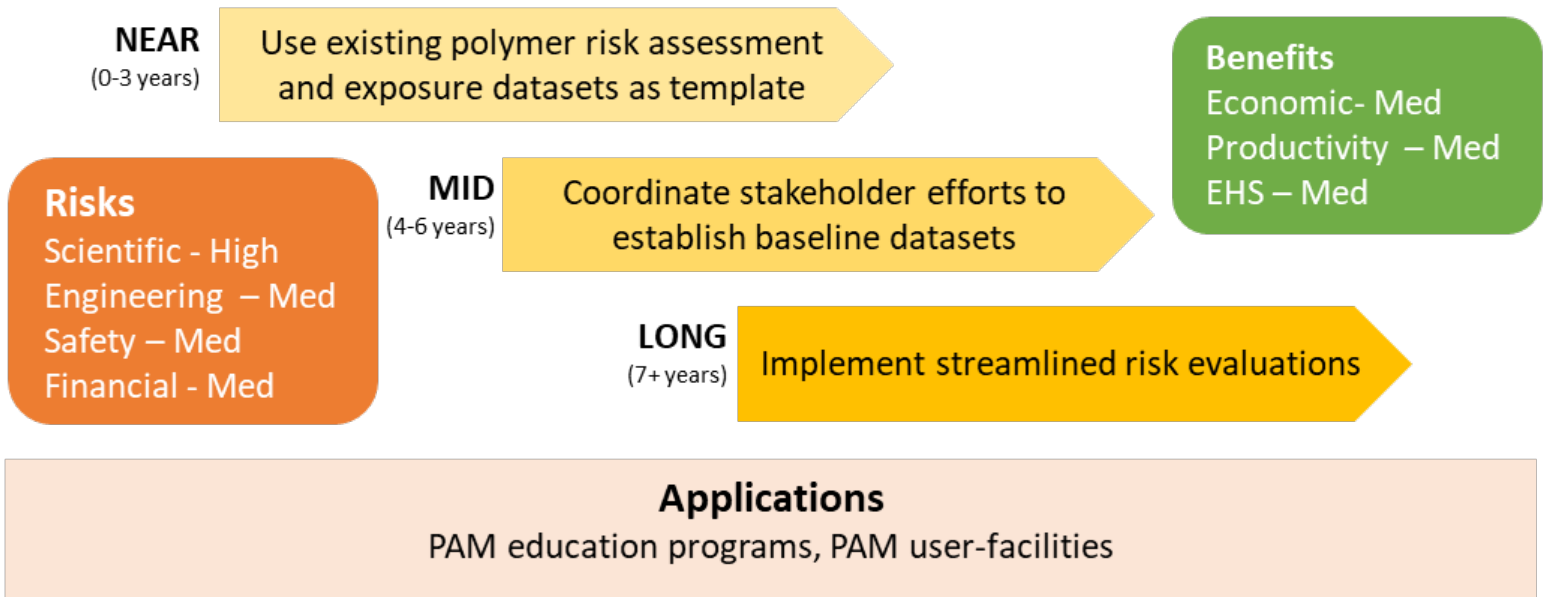

ROADMAPPED SOLUTION: DEFINE MINIMAL DATASET FOR RISK ASSESSMENT, EXPOSURE

Originating from Panel IV: Health, Safety and Regulation in AM 


\section{Priority \\ MEDIUM \\ Develop Framework for Product Stewardship}

RRD: EHS Reg. for

Effective PAM Adoption

\section{Objectives}

- Require responsible development of new materials and products

- Enhance public confidence in technology and products by establishing life cycle assessments

- Support regulatory frameworks via multi-stakeholder collaboration

\begin{tabular}{|c|c|c|c|}
\hline \multicolumn{4}{|c|}{ Partners } \\
\hline Industry (I) & \multicolumn{2}{|c|}{ Academic (A) } & Federal (F) \\
\hline $\begin{array}{c}\text { Resin formulators, } \\
\text { printer OEMs, trade } \\
\text { organizations, and end users }\end{array}$ & \multicolumn{2}{|c|}{$\begin{array}{l}\text { Toxicologist and environmental } \\
\text { scientists }\end{array}$} & $\begin{array}{c}\text { Science and regulatory } \\
\text { agencies, high-risk research } \\
\text { programs }\end{array}$ \\
\hline \multicolumn{2}{|c|}{$\begin{array}{l}\text { Current Efforts (2020) } \\
\text { - } \text { Circular economy efforts to establish } \\
\text { markets to deploy sustainability (I \& F) } \\
\text { - } \text { Resin and resin precursor reuse under } \\
\text { study (I, A, \& F) } \\
\text { - } \quad \text { Upcycling/end of life considerations being } \\
\text { researched (I \& F) }\end{array}$} & $\begin{array}{l}\text { - Industry b } \\
\text { - Clearly de } \\
\text { capabilitie } \\
\text { - Decouplin } \\
\text { from healt } \\
\text { - Substantia }\end{array}$ & $\begin{array}{l}\text { Challenges } \\
\text { y-in across the supply chain } \\
\text { ning product stewardship } \\
\text { and needs } \\
\text { workplace exposures needs } \\
\text { effects research } \\
\text { R\&D required }\end{array}$ \\
\hline
\end{tabular}

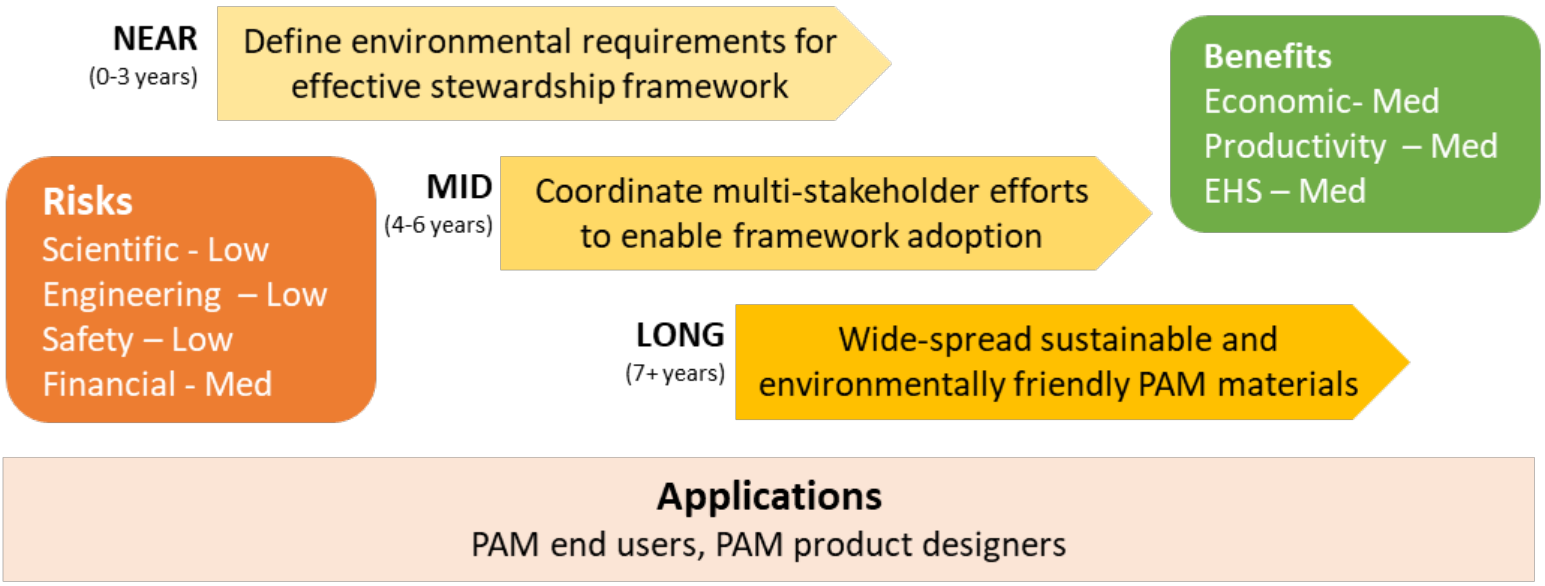

ROADMAPPED SOLUTION: DEVELOP FRAMEWORK FOR PRODUCT STEWARDSHIP

Originating from Panel IV: Health, SAfety ANd Regulation in AM 


\section{Priority \\ LOW \\ Design Education Material on Green \\ Photopolymers}

RRD: EHS Reg. for Effective PAM Adoption

\section{Objectives}

- Expand knowledge base with new workforce training

- Raise PAM value as a manufacturing technology via sustainable material investment

- Develop next generation PAM opportunities

\begin{tabular}{|c|c|c|}
\hline \multicolumn{3}{|c|}{ Partners } \\
\hline Industry (I) & Academic (A) & Federal (F) \\
\hline $\begin{array}{l}\text { Resin formulators, } \\
\text { end users, and pre-competitive } \\
\text { consortia members }\end{array}$ & Chemists and material scientists & $\begin{array}{l}\text { Education-focused programs, } \\
\text { circular economy agencies }\end{array}$ \\
\hline
\end{tabular}

\section{Current Efforts (2020)}

- Concerted but uncoordinated sustainable materials research (I, A, \& F)

- Sustainable materials management grants (F)

- Environmental education grants (F)

\section{Challenges}

- Speed of market changes, requirements

- Transition from self-learning to formal training and academic models

- Lack of business case to address education and training needs

- Student embedding in industry (all levels)
NEAR

(0-3 years)
Benefits

Economic- Med

Productivity - Low

EHS - Med

\section{Risks}

Scientific - Low

Engineering - Low

Safety - Low

Financial - Med

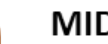

(4-6 years)
Develop curriculum (trade organizations, universities)

\section{LONG Establish accredited educaton programs}

\section{Applications}

Streamlined PAM via reduced waste, personalized medicine

ROADMAPPED SOLUTION: DESIGN EDUCATION MATERIAL ON GREEN PHOTOPOLYMERS

Originating from PANel I: SUStainable, Hybrid, AND Emerging MAterial Systems 


\section{RRD: Industry Collaborations to Enhance PAM Utility}

The Roadmapped Solutions under the Advanced Computing for Materials Discovery and Process modelling Recommended Research Direction focus on the requisite computational needs to expedite new material development and optimize printing. Many science and engineering challenges must be addressed for PAM to achieve its full potential. The rapid pace of innovation by PAM industry requires alignment amongst stakeholders to ensure priority research needs are being addressed. Fundamental and applied research investments must be balanced to ensure short-term and long-term successes. Listed below are each of the each roadmapped solutions with the corresponding panel in which they originated.

High Priority Roadmapped Solutions:

\section{Translate commercial needs into R\&D efforts}

- Improve industry coordination in communicating research needs to research community

- Create communication structure between academia, government, and industry to enable pre-competitive research sharing

- Panel III: Industry Applications and Developing Markets

\section{Support collaborations between small- and large-scale PAM industry adopters}

- Develop models for intellectual property protection and licensing between private and public sectors

- Improve technology transfer to match PAM capabilities to industry needs

- Entrepreneurship and technology transfer training into academic programs to support small PAM manufacturing companies

- Panel III: Industry Applications and Developing Markets

Medium Priority Roadmapped Solutions:

Advance manufacturing capabilities in PAM

- Create programs to sponsor facilities for testing and shared use production for PAM implementation in novel markets

- Use such facilities to expedite PAM resin formulation, resin testing, and pilot programs

- Panel III: Industry Applications and Developing Markets

Build value proposition to meet customer needs

- Clearly define utility of PAM for a variety of application spaces

- Prioritize commercial needs and translate these areas of study into research funding investments

- Panel I: Sustainable, Hybrid, and Emerging Material Systems

Lower Priority Roadmapped Solutions:

\section{Pursue precision engineering and manufacturing}

- Highlight PAM's unique capacity to realize precision engineering and manufacturing via versatile materials and print processes

- Create programs to elevate data science value among PAM manufacturers

- Panel V: Moonshot Ideas in PAM 


\section{Objectives}

- Build framework to integrate industry needs with an academic funding mechanism

- Establish implementable results-driven capabilities for new PAM applications

\begin{tabular}{|c|c|c|}
\hline & \multicolumn{2}{|c|}{ Partners } \\
\hline Industry (I) & Academic (A) & Federal (F) \\
\hline $\begin{array}{c}\text { Resin formulators, } \\
\text { printer OEMs, and end users }\end{array}$ & $\begin{array}{c}\text { Material scientists, chemists, } \\
\text { and optical scientists }\end{array}$ & $\begin{array}{c}\text { Funding and R\&D agencies, } \\
\text { open-user facilities }\end{array}$ \\
\hline
\end{tabular}

\section{Current Efforts (2020)}

- Multi-stakeholder workshops (I, A, \& F)

- Trade organization stakeholder engagement efforts (webinars, conferences, etc.) (I)

\section{Challenges}

- Consensus building of a research agenda

- Coordination between funding agencies, industry and academics

- Getting new developments into supply chain

- Selling new PAM products
NEAR

(0-3 years)

Engage PAM stakeholders to develop cohesive strategy for translational R\&D

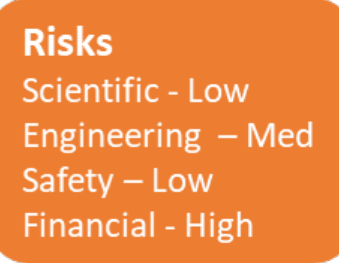

Establish federal and/or industry funded R\&D programs

\section{LONG Translate R\&D programs into start-up} $(7+$ years $)$ and mainstream manufacturing efforts

\section{Benefits}

Economic- High

Productivity - Med

EHS - Med

\section{Applications}

PAM system development, PAM end users, resin formulation

ROADMAPPED SOLUTION: TRANSLATE COMMERCIAL NEEDS INTO R\&D EFFORTS

ORIGINATING FROM PANEL III: INDUSTRY APPLICATIONS AND DEVELOPING MARKETS 


\section{Priority \\ HIGH \\ Support Collaborations Between Small- and Large-Scale PAM Industry Adopters}

RRD: Ind. Collab. to Enhance PAM Utility

\section{Objectives}

- Grow proof-of-concept capabilities and connect scalability of production to design

- Support start-ups by young entrepreneurs

- Incentivize new product divisions in larger manufacturers

\begin{tabular}{|c|c|c|}
\hline \multicolumn{3}{|c|}{ Partners } \\
\hline Industry (I) & Academic (A) & Federal (F) \\
\hline $\begin{array}{c}\text { Resin formulators, } \\
\text { printer OEMs, trade } \\
\text { organizations, and end users }\end{array}$ & Consortia members & $\begin{array}{c}\text { Manufacturing centers and } \\
\text { funding agencies }\end{array}$ \\
\hline
\end{tabular}

\section{Current Efforts (2020)}

- America Makes research opportunities for advanced manufacturing (F)

- Manufacturing Extension Partnership centers to drive public-private partnerships (I \& F)

\section{Challenges}

- IP and proprietary issues for industry

- Safety education and training on new instruments and models

- Agreement on strategic investments for shared use capabilities

- Robust testing procedures

\section{NEAR \\ (0-3 years)

$$
\begin{aligned}
& \text { Organize government-facilitated } \\
& \text { industry-wide PAM coalition }
\end{aligned}
$$

\section{Risks}

Scientific - Med

Engineering - Med

Safety - Low

Financial - High

$$
\begin{array}{r}
\text { MID Use regular meetings to connect and } \\
\text { exchange recent advances }
\end{array}
$$

Benefits

Economic- Med

Productivity - High

EHS - Low

$$
\begin{array}{r}
\text { LONG Increased entrepreneurship and } \\
\text { (7+years) } \\
\text { company outgrowth }
\end{array}
$$

\section{Applications}

Innovative cross-industry PAM solutions, streamlined PAM optimization

ROADMAPPED SOLUTION: SUPPORT COLLABORATIONS BETWEEN SMALL- AND LARGE-SCALE PAM INDUSTRY ADOPTERS

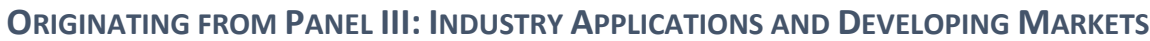




\section{Priority
MEDIUM $\quad$ Advance Manufacturing Capabilities in PAM $\quad \begin{gathered}\text { RRD: Ind. Collab. } \\ \text { to Enhance PAM } \\ \text { Utility }\end{gathered}$}

\section{Objectives}

- Create programs for shared facilities for testing, pilots, and scale-up

- Establish advanced manufacturing equipment, i.e., hardware and software

- Improve speed and process controls with technological investment

\begin{tabular}{|c|c|c|}
\hline \multicolumn{2}{|c|}{ Partners } \\
\hline Industry (I) & Academic (A) & Federal (F) \\
\hline $\begin{array}{c}\text { Resin formulators, } \\
\text { printer OEMs, and end users }\end{array}$ & $\begin{array}{c}\text { Material scientists, chemists, } \\
\text { optical engineers, and } \\
\text { manufacturing experts }\end{array}$ & $\begin{array}{c}\text { Funding and R\&D agencies, } \\
\text { manufacturing programs }\end{array}$ \\
\hline
\end{tabular}

\section{Current Efforts (2020)}

- Novel PAM printers coming to market (I, A, \& F)

- Resin chemistries with faster reaction kinetics (I \& A)

- Multiple, low-cost printers (I)

- Optimized post processing (I \& A)

\section{Challenges}

- Consensus building of engineering, process and technology needs

- Cost, investments, and industry buy-in

- Education and training of new systems

- Software and hardware developments

- Light source needs

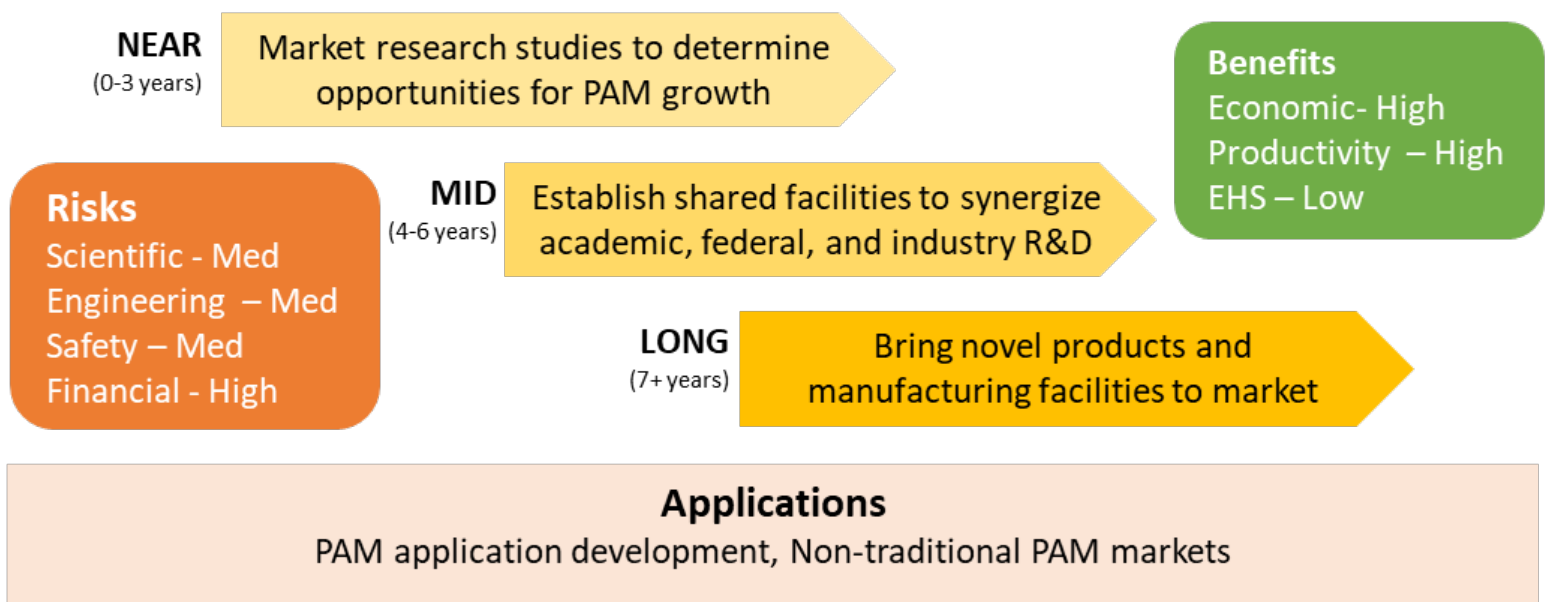

ROADMAPPED SOLUTION: AdVANCE MANUFACTURING CAPABILITIES IN PAM

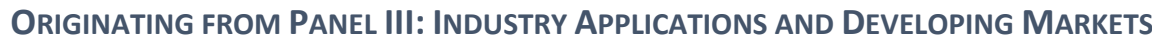




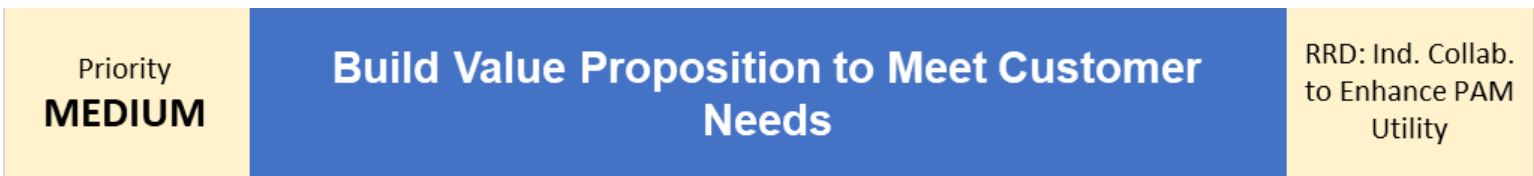

\section{Objectives}

- Grow capabilities to uniformly define, evaluate, measure, and build value

- Develop open source database of case study successes

\begin{tabular}{|c|c|c|}
\hline & Partners & \\
\hline Industry (I) & Academic (A) & Federal (F) \\
\hline $\begin{array}{c}\text { Printer OEMs, trade } \\
\text { organizations, and end users }\end{array}$ & $\begin{array}{c}\text { Business schools with } \\
\text { technology focus }\end{array}$ & $\begin{array}{l}\text { R\&D agencies, technology } \\
\text { development offices, and } \\
\text { manufacturing programs }\end{array}$ \\
\hline
\end{tabular}

\section{Current Efforts (2020)}

- Targeted marketing campaigns (I \& F)

- Open-source, crowdsourcing of optimal printing conditions (I, A, \& F)

- All-hands stakeholder conferences (I, A, \& F)

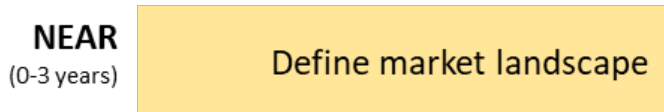

\section{Risks \\ Scientific - Med \\ Engineering - Med \\ Safety - Low \\ Financial - High}

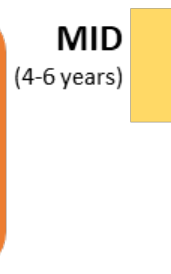

Tools to evaluate opportunities

\section{Challenges}

- Business focus on features and products rather than process and technology

- Game-changing opportunities cause disruption to current processes or environments

- Widespread adoption of new materials

\section{Applications \\ PAM adoption across industries, resin formulation, PAM process design}

ROADMAPPED SOLUTION: BUILD VALUE PROPOSITION TO MEET CUSTOMER NEEDS

Originating from Panel I: Sustainable, Hybrid, AND Emerging Material Systems 


\section{Objectives}

- Increased precision printing with In-line measurement capabilities at nanometer scale

- Understand process-structure (engineering properties)

- Improved safety, performance, and product longevity

\begin{tabular}{|c|c|c|}
\hline & \multicolumn{2}{|c|}{ Partners } \\
\hline Industry (I) & Academic (A) & Federal (F) \\
\hline $\begin{array}{c}\text { Resin formulators, } \\
\text { printer OEMs, and end users }\end{array}$ & $\begin{array}{c}\text { Mechanical engineers, } \\
\text { manufacturing and process } \\
\text { specialists }\end{array}$ & $\begin{array}{c}\text { R\&D agencies and } \\
\text { manufacturing programs }\end{array}$ \\
\hline
\end{tabular}

\section{Current Efforts (2020)}

- Micro-stereolithography and two-photo printing (I, A, \& F)

- Nano-micro characterization applied to print property engineering ( $A \& F)$

\section{Challenges}

- Integrating materials science with precision engineering

- Tolerance and scalability needs

- Supply chain buy in

- Metrology at the nanoscale

- Lack of test facilities

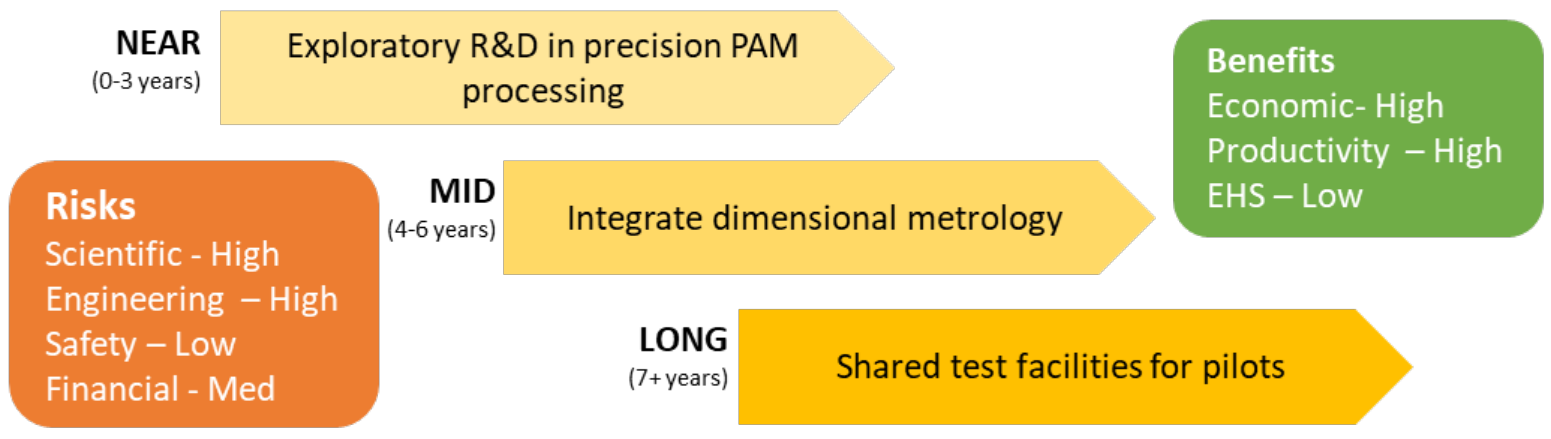

\section{Applications}

Personalized medicine, novel meta-material development

ROAdMAPPED SOlUtion: PURSUE PRECISION ENGINEERING AND MANUfACtURING

ORIGINATING FRom PANel V: MOONSHOt IDEAS IN PAM 


\section{Appendix A: List of Participants}

*PAM Workshop 2019 Planning Committee members

Abeer Alzahrani, Whip Mix Corporation Aditya Balasubramanian, polySpectra Stephanie Benight, Tactile Materials Solutions Karine Blandel, In-Vision Technologies USA Inc.

Michael Brady, 3D Systems Inc.

Tobin Brown, NIST

Tom Bugnitz, Manufacturer's Edge

Yan Chen, Align Technology

Anthony Clay, US Army Research Laboratory

*Gary Cohen, RadTech

Denis Cormier, Rochester Institute of

Technology

* Neil Cramer, Colorado Photopolymer

Solutions

Rachel Davis, Azul 3D

Marla Dowell, NIST

Gregory Ellson, Inkbit

* Mickey Fortune Jr., RadTech

Robert Gafvert, Carbon, Inc.

Edward Garboczi, NIST

Alicia Gibson, MacDermid Graphics Solutions

Andrew Harbourne, Harbourne Consulting

Services LLC

John Hergert, CU Boulder

*Callie Higgins, NIST, Chair

Jacob Hutfles, University of Colorado

*Mike Idacavage, Colorado Photopolymer

Solutions

Rakesh Jain, polySpectra

Jedediah Kallen-Brown, CU Boulder

Hisao Kato, Toagosei America

John Kawola, BMF Precision

Kenji Kikuta, Kowa American Corp

Marvin Kilgo, Radicle Fabrication LLC

*Jason Killgore, NIST, Chair

Kangmin Kim, University of Colorado Boulder

Jeffrey Klang, Sartomer

Anthony Kotula, NIST
Rajan Kumar, Ateios

John La Scala, U.S. Army Combat Capabilities

Development Command's Army Research

Laboratory

Simon Lancaster-Larocque, Apple

Chunhua Li, Align Technology

Chong Li, Meixin Technology, Inc.

Spencer Loveless, Dustless Technologies

Robert MacCurdy, CU Boulder

Veruska Malave, NIST

Esteban Marin Guillen, Heraeus Noblelight

North America LLC

Annabel Marruffo, Vartega Inc.

Joselle McCracken, CU Boulder

Thomas McKeag, Berkeley Center for Green

Chemistry

Robert McLeod, CU Boulder

Alexandre Mejiritski, Spectra Photopolymers

*Cameron Miller, NIST

Archish Muralidharan, CU Boulder

Kevin Perez, nTopology

John Perkins, NIST

Justin Poelma, Carbon, Inc.

*Dianne Poster, NIST

Charles Rackson, CU Boulder

William Richardson, Anderson \& Vreeland

David Robitaille, Dymax Oligomers \& Coatings

Eric Romano, DSM

Gary Roth, National Institute for Occupational

Safety and Health

Samuel Ruben, Mighty Buildings, Inc

Jatinder Sampathkumar, CU Boulder

Manilal Savla, Savla Associates

Parag Shah, University of Colorado

Pratik Shah, Adaptive3D

Paul Share, Advanced Materials Design

James Shin, Align Technology

Maxim Shusteff, Lawrence Livermore National

Laboratory

Nicholas Singstock, CU Boulder

Jeremy Smith, Nagase 
Jeffrey Stansbury, University of Colorado School of Dental Medicine

Alexandria Stanton, U.S. EPA

Will Tashman, Uncountable

Treye Thomas, US CPSC

Scott Turner, 3D Systems Inc.

Asais Uzcategui, CU Boulder

Tia Vialva, 3D Printing Industry

Travis Walker, South Dakota School of Mines

\& Technology
David Walker, Azul3D

Marlon Walker, NIST MEP

Xiaojiang Wang, Ford Motor Company

Christopher Williams, DREAMS Laboratory at

Virginia Tech

Charles Wood, Fast Radius, Inc

Youyuan Wu, IGM Resins

Ima Yaghoubi Rad, Sepanta, Inc.

Kai Yang, Wikoff Color Corporation

Maximilian Zieringer, Formlabs 


\section{Appendix B: Agenda}

October 29, 2019

7:30am Registration/Check-In

8:30 am Opening Remarks \& NIST Welcome

9:00 am Keynote Presentation: Frank Gayle, NIST

9:30 am Stakeholder Leader Perspectives I

10:15 am Break

10:30 am Stakeholder Leader Perspectives II

11:30 pm Panel I: Sustainable, Hybrid, and Emerging Material Systems

$12: 30$ pm Lunch

2:00 pm Panel II: AM-specific characterization

3:00 pm Panel III: Industry Applications and Developing Markets

4:00 pm Reception

October 30, 2019

9:00 am Day 1 Take-aways \& Discussion

10:00 am Panel IV: Health, Safety, and Regulatory

11:00 am Panel V: AM Moonshots

12:00 pm Lunch and Closing Remarks

2:00 pm Lab Tours

2:00 pm - NIST and CPS

3:00 pm - 3D Systems Healthcare 